\title{
Symmetries of Baryons and Mesons*
}

\author{
Murray Gell-ManN \\ California Institute of Technology, Pasadena, California \\ (Received March 27, 1961; revised manuscript received September 20, 1961)
}

The system of strongly interacting particles is discussed, with electromagnetism, weak interactions, and gravitation considered as perturbations. The electric current $j_{\alpha}$, the weak current $J_{\alpha}$, and the gravitational tensor $\theta_{\alpha \beta}$ are all well-defined operators, with finite matrix elements obeying dispersion relations. To the extent that the dispersion relations for matrix elements of these operators between the vacuum and other states are highly convergent and dominated by contributions from intermediate one-meson states, we have relations like the Goldberger-Treiman formula and universality principles like that of Sakurai according to which the $\rho$ meson is coupled approximately to the isotopic spin. Homogeneous linear dispersion relations, even without subtractions, do not suffice to fix the scale of these matrix elements; in particular, for the nonconserved currents, the renormalization factors cannot be calculated, and the universality of strength of the weak interactions is undefined. More information than just the dispersion relations must be supplied, for example, by field-theoretic models; we consider, in fact, the equal-time commutation relations of the various parts of $j_{4}$ and $J_{4}$. These nonlinear relations define an algebraic system (or a group) that underlies the structure of baryons and mesons. It is suggested that the group is in fact $U(3) \times U(3)$, exemplified by the symmetrical Sakata model. The Hamiltonian density $\theta_{44}$ is not completely invariant under the group; the noninvariant part transforms according to a particular

\section{INTRODUCTION}

$I^{\prime}$ $\mathrm{N}$ connection with the system of strongly interacting particles, there has been a great deal of discussion of possible approximate symmetries, ${ }^{1}$ which would be violated by large effects but still have some physical consequences, such as approximate universality of meson couplings, approximate degeneracy of baryon or meson supermultiplets, and "partial conservation" of currents for the weak interactions.

In this article we shall try to clarify the meaning of such possible symmetries, for both strong and weak interactions. We shall show that a broken symmetry, even though it is badly violated, may give rise to certain exact relations among measurable quantities. Furthermore, we shall suggest a particular symmetry group as the one most likely to underlie the structure of the system of baryons and mesons.

We shall treat the strong interactions without approximation, but consider the electromagnetic, weak, and gravitational interactions only in first order.

The electromagnetic coupling is described by the matrix elements of the electromagnetic current operator $e j_{\alpha}(x)$. Likewise, the gravitational coupling is specified by the matrix elements of the stress-energy-momentum

* Research supported in part by U. S. Atomic Energy Commission and Alfred P. Sloan Foundation. A report of this work was presented at the La Jolla Conference on Strong and Weak Interactions, June, 1961.

"For example, see the "global symmetry" scheme of M. GellMann, Phys. Rev. 106, 1296 (1957) and J. Schwinger, Ann. Phys. 2, 407 (1957). representation of the group; it is possible that this information also is given correctly by the symmetrical Sakata model. Various exact relations among form factors follow from the algebraic structure. In addition, it may be worthwhile to consider the approximate situation in which the strangeness-changing vector currents are conserved and the Hamiltonian is invariant under $U(3)$; we refer to this limiting case as "unitary symmetry." In the limit, the baryons and mesons form degenerate supermultiplets, which break up into isotopic multiplets when the symmetry-breaking term in the Hamiltonian is "turned on." The mesons are expected to form unitary singlets and octets; each octet breaks up into a triplet, a singlet, and a pair of strange doublets. The known pseudoscalar and vector mesons fit this pattern if there exists also an isotopic singlet pseudoscalar meson $\chi^{0}$. If we consider unitary symmetry in the abstract rather than in connection with a field theory, then we find, as an attractive alternative to the Sakata model, the scheme of Ne'eman and Gell-Mann, which we call the "eightfold way"; the baryons $N, \Lambda, \Sigma$, and $\Xi$ form an octet, like the vector and pseudoscalar meson octets, in the limit of unitary symmetry. Although the violations of unitary symmetry must be quite large, there is some hope of relating certain violations to others. As an example of the methods advocated, we present a rough calculation of the rate of $K^{+} \rightarrow \mu^{+}+\nu$ in terms of that of $\pi^{+} \rightarrow \mu^{+}+\nu$.

tensor $\theta_{\alpha \beta}(x)$, particularly the component $\theta_{44}=H$, the Hamiltonian density.

The weak interactions of baryons and mesons with leptons are assumed to be given (ignoring possible nonlocality) by the interaction term ${ }^{2}$

$$
G J_{a}^{\dagger} J_{\alpha}{ }^{(l)} / \sqrt{2}+\text { H.c., }
$$

where the leptonic weak current $J_{\alpha}{ }^{(l)}$ has the form

$$
J_{\alpha}{ }^{(l)}=i \bar{\nu} \gamma_{\alpha}\left(1+\gamma_{5}\right) e+i \bar{\nu} \gamma_{\alpha}\left(1+\gamma_{5}\right) \mu .
$$

We shall refer to $J_{\alpha}(x)$ as the weak current of baryons and mesons. Its matrix elements specify completely the weak interactions with leptons.

It is possible that the full weak interaction may be given simply by the term

$$
G\left(J_{\alpha}+J_{\alpha}{ }^{(l)}\right)^{\dagger}\left(J_{\alpha}+J_{\alpha}{ }^{(l)}\right) / \sqrt{2},
$$

although this form provides no explanation of the approximate rule $|\Delta \mathbf{I}|=\frac{\mathbf{1}}{\mathbf{2}}$ in the nonleptonic decays of strange particles. If we can find no dynamical explanation of the predominance of the $|\Delta \mathbf{I}|=\frac{1}{2}$ amplitude in these decays, we may be forced to assume that in addition to (1.3) there is a weak interaction involving the product

$$
G L_{\alpha}^{\dagger} L_{\alpha} / \sqrt{2}
$$

of charge-retention currents (presumably not involving leptons); or else we may be compelled to abandon (1.3)

${ }^{2}$ We use $\hbar=c=1$. The Lorentz index $\alpha$ takes on the values $1,2,3,4$. For each value of $\alpha$, the Dirac matrix $\gamma_{\alpha}$ is Hermitian; so is the matrix $\gamma_{5}$. 
altogether. In any case, we shall define the weak current $J_{\alpha}$ by the coupling to leptons.

We shall assume microcausality and hence the validity of dispersion relations for the matrix elements of the various currents and densities. In addition, we shall sometimes require the special assumption of highly convergent dispersion relations.

Our description of the symmetry group for baryons and mesons is most conveniently given in the framework of standard field theory, where the Lagrangian density $L$ of the strong interactions is expressed as a simple function of a certain number of local fields $\psi(x)$, which are supposed to correspond to the "elementary" baryons and mesons. Recently this type of formalism has come under criticism ${ }^{3}$; it is argued that perhaps none of the strongly interacting particles is specially distinguished as "elementary," that the strong interactions can be adequately described by the analyticity properties of the $S$ matrix, and that the apparatus of field theory may be a misleading encumbrance.

Even if the criticism is justified, the field operators $j_{\alpha}(x), \theta_{\alpha \beta}(x)$, and $J_{\alpha}(x)$ may still be well defined (by all their matrix elements, including analytic continuations thereof) and measurable in principle by interactions with external electromagnetic or gravitational fields or with lepton pairs. Since the Hamiltonian density $H$ is a component of $\theta_{\alpha \beta}$, it can be a physically sensible quantity.

In order to make our description of the symmetry group independent of the possibly doubtful details of field theory, we shall phrase it ultimately in terms of the properties of the operators $H, j_{\alpha}$, and $J_{\alpha}$. In introducing the description, however, we shall make use of fieldtheoretic models. Moreover, in describing the behavior of a particular group, we shall refer extensively to a special example, the symmetrical Sakata model of Ohnuki et al., ${ }^{4}$ Yamaguchi, ${ }^{5}$ and Wess. ${ }^{6}$

The order of presentation is as follows: We treat first the hypothesis of highly convergent dispersion relations for the matrix elements of currents; and we show that the notion of a meson being coupled "universally" or coupled to a particular current or density means simply that the meson state dominates the dispersion relations for that current or density at low momenta. Next we discuss the universality of strength of the currents themselves; evidently it cannot be derived from homogeneous linear dispersion relations for the matrix elements of the currents. We show that equal-time commutation relations for the currents fulfill this need (or most of it), and that, in a wide class of model field

${ }^{3}$ G. F. Chew, Talk at La Jolla Conference on Strong and Weak Interactions, June, 1961 (unpublished).

${ }^{4}$ M. Ikeda, S. Ogawa, and Y. Ohnuki, Progr. Theoret. Phys. (Kyoto) 22, 715 (1959); Y. Ohnuki, Proceedings of the 1960 Annual International Conference on High-Energy Physics at Rochester (Interscience Publishers, Inc., New York, 1960).

5 Y. Yamaguchi, Progr. Theoret. Phys. (Kyoto) Suppl. No. 11, 1 (1959).

${ }^{6} \mathrm{~J}$. Wess, Nuovo cimento 10, 15 (1960). theories, these commutation rules are simple and reflect the existence of a symmetry group, which underlies the structure of the baryon-meson system even though some of the symmetries are badly violated. We present the group properties in an abstract way that does not involve the details of field theory.

Next, it is asked what group is actually involved. The simplest one consistent with known phenomena is the one suggested. It is introduced, for clarity, in connection with a particular field theory, the symmetrical Sakata model, in which baryons and mesons are built up of fundamental objects with the properties of $n, p$, and $\Lambda$. For still greater simplicity, we discuss first the case in which $\Lambda$ is absent.

We then return to the question of broken symmetry in the strong interactions and show how some of the symmetries in the group, if they are not too badly violated, would reveal themselves in approximately degenerate supermultiplets. In particular, there should be "octets" of mesons, each consisting of an isotopic triplet with $S=0$, a pair of doublets with $S= \pm 1$, and a singlet with $S=0$. In the case of pseudoscalar mesons, we know of $\pi, K$, and $\bar{K}$; these should be accompanied by a singlet pseudoscalar meson $\chi^{0}$, which would decay into $2 \gamma, \pi^{+}+\pi^{-}+\gamma$, or $4 \pi$, depending on its mass.

In Sec. VIII, we propose, as an alternative to the symmetrical Sakata model, another scheme with the same group, which we call the "eightfold way." Here the baryons, as well as mesons, can form octets and singlets, and the baryons $N, \Lambda, \Sigma$, and $\Xi$ are supposed to constitute an approximately degenerate octet.

In Sec. IX, some topics are suggested for further investigation, including the possibility of high energy limits in which non-conserved quantities become conserved, and we give, as an example of methods suggested here, an approximate calculation of the rate of $K^{+} \rightarrow \mu^{+}+\nu$ decay from that of $\pi^{+} \rightarrow \mu^{+}+\nu$ decay.

\section{MESONS AND CURRENTS}

To introduce the connection between meson states and currents or densities, let us review the derivation ${ }^{7}$ of the Goldberger-Treiman relation ${ }^{8}$ among the charged pion decay amplitude, the strength of the axial vector weak interaction in the $\beta$ decay of the nucleon, and the pion-nucleon coupling constant.

The axial vector term in $J_{\alpha}$ with $\Delta S=0,|\Delta \mathbf{I}|=1$, $G P=-1$, can be written as $P_{1 \alpha}+i P_{2 \alpha}$, where $\mathbf{P}_{\alpha}$ is an axial vector current that transforms like an isotopic vector. We have, for nucleon $\beta$ decay,

$$
\left\langle N\left|\mathbf{P}_{\alpha}\right| N\right\rangle=\bar{u}_{f}\left[i \gamma_{\alpha} F_{\mathrm{ax}}(s)+k_{\alpha} \beta(s)\right] \gamma_{5}(\tau / 2) u_{i},
$$

where $u_{i}$ and $u_{f}$ are the initial and final spinors, $k_{\alpha}$ is the four-momentum transfer, and $s=-k^{2}=-k_{\alpha} k_{\alpha}$. At

${ }^{7}$ J. Bernstein, S. Fubini, M. Gell-Mann, and W. Thirring, Nuovo cimento 17, 757 (1960). See also Y. Nambu, Phys. Rev. Letters 4,380 (1960); and Chou Kuang-Chao, Soviet Phys.-JETP 12, 492 (1961).

${ }^{8}$ M. Goldberger and S. Treiman, Phys. Rev. 110, 1478 (1958) 
$s=0$ we have just

$$
F_{\mathrm{ax}}(0)=-G_{A} / G
$$

the axial vector renormalization constant.

The axial vector current is not conserved; its divergence $\partial_{\alpha} \mathbf{P}_{\alpha}$ has the same quantum numbers as the pion $\left(J=0^{-}, I=1\right)$. Between nucleon states we have

$$
\left\langle N\left|\partial_{\alpha} \mathbf{P}_{\alpha}\right| N\right\rangle=\bar{u}_{f} i \gamma_{5}(\tau / 2) u_{i}\left[2 m_{N} F_{\mathrm{ax}}(s)+s \beta(s)\right] .
$$

We may compare this matrix element with that between the vacuum and a one-pion state

$$
\left\langle 0\left|\partial_{\alpha} \mathbf{P}_{\alpha}\right| \pi\right\rangle=m_{\pi}^{2}\left(2 f_{\pi}\right)^{-1} \boldsymbol{\phi}
$$

where $\boldsymbol{\phi}$ is the pion wave function and the constant $f_{\pi}$ (or at least its square) may be measured by the rate of $\pi^{+} \rightarrow \mu^{+}+\nu$ :

$$
\Gamma_{\pi}=G^{2} m_{\pi} m_{\mu}^{2}\left(1-m_{\mu}^{2} / m_{\pi}^{2}\right)^{2}\left(f_{\pi}^{2} / 4 \pi\right)^{-1}\left(6+\pi^{2}\right)^{-1} .
$$

It is known that the matrix element (2.3) has a pole at $s=m_{\pi}{ }^{2}$ corresponding to the virtual emission of a pion that undergoes leptonic decay. The strength of the pole is given by the product of $m_{\pi}^{2} / f_{\pi}$ and the pionnucleon coupling constant $g_{N N \pi}$. If we assume that the expression in brackets vanishes at large $s$, we have an unsubtracted dispersion relation for it consisting of the pole term and a branch line beginning at $\left(3 m_{\pi}\right)^{2}$, the next lowest mass that can be virtually emitted:

$$
\begin{aligned}
& 2 m_{N} F_{\mathrm{ax}}(s)+s \beta(s)=\left(g_{N N \pi} / f_{\pi}\right) m_{\pi}^{2}\left(m_{\pi}^{2}-s\right)^{-1} \\
& +\int \sigma_{\mathrm{ax}}\left(M^{2}\right) M^{2} d M^{2}\left(M^{2}-s-i \epsilon\right)^{-1} .
\end{aligned}
$$

At $s=0$, we have, using (2.2), the sum rule

$$
2 m_{N}\left(-G_{A} / G\right)=g_{N N \pi} / f_{\pi}+\int \sigma_{\operatorname{ax}}\left(M^{2}\right) d M^{2} .
$$

Now if the dispersion relation (2.6) is not only convergent but dominated at low $s$ by the term with the lowest mass, then we have the approximate GoldbergerTreiman relation

$$
2 m_{N}\left(-G_{A} / G\right) \approx g_{N N \pi} / f_{\pi},
$$

which agrees with experiment to within a few percent.

The success of the relation suggests that other matrix elements of $\partial_{\alpha} \mathbf{P}_{\alpha}$ may also obey unsubtracted dispersion relations dominated at low $s$ by the one-pion term. For example, if we consider the matrix element between $\Lambda$ and $\Sigma$, we should arrive at the relation

$$
\left(m_{\Lambda}+m_{\Sigma}\right)\left(-G_{\Lambda} \Lambda \Sigma / G\right) \approx g_{\Lambda \Sigma \pi} / f_{\pi},
$$

if $\Lambda$ and $\Sigma$ have the same parity, or an analogous relation if they have opposite parity.

If such a situation actually obtains, then it may be said that the pion is, to a good approximation, coupled "universally" to the divergence of the axial vector current. To calculate any $g$ approximately, we multiply the universal constant $f_{\pi}$, the sum of the initial and final masses, and the renormalization factor for the axial vector current.

Now let us turn to the case of a current that is conserved, say the isotopic spin current $\mathfrak{J}_{\alpha}$ with quantum numbers $J=1^{-}, I=1$. Acting on the vacuum, the operator $\mathfrak{\Im}_{\alpha}$ does not lead to any stable one-meson state, but it does lead to the unstable vector meson state $\rho$ at around $750 \mathrm{Mev}$, which decays into $2 \pi$ or $4 \pi$. For simplicity, let us ignore the rather large width $\left(\Gamma_{\rho} \sim 100\right.$ Mev) of the $\rho$ state and treat it as stable. The mathematical complications resulting from the instability are not severe and have been discussed elsewhere. ${ }^{9,10}$

In place of (2.4), then, we have the definition

$$
\left\langle 0\left|\Im_{\alpha}\right| \rho\right\rangle=m_{\rho}^{2}\left(2 \gamma_{\rho}\right)^{-1} \boldsymbol{\phi}_{\alpha},
$$

of the constant $\gamma_{\rho}$, where $\phi_{\alpha}$ is the wave function of the $\rho$ meson. In place of (2.1) or (2.3), we consider the matrix element between nucleon states of the isotopic spin current:

$\left\langle N\left|\mathfrak{J}_{\alpha}\right| N\right\rangle=\bar{u}_{f} i \gamma_{\alpha}(\tau / 2) u_{i} F_{1}^{V}(s)$ t-magnet ic term,

where $F_{1} V(s)$ is the familiar isovector form factor of the electric charge of the nucleon, since the electromagnetic current has the form

$$
j_{\alpha}=\mathfrak{\Im}_{3 \alpha}+\text { isoscalar term. }
$$

If we continue to ignore the width of $\rho$, we get a dispersion relation like $(2.6)$ with a pole term at $m_{\rho}^{2}$ :

$$
\begin{aligned}
& F_{1}^{V}(s)=\left(\gamma_{N N \rho} / \gamma_{\rho}\right) m_{\rho}^{2}\left(m_{\rho}^{2}-s\right)^{-1} \\
& \quad+\int \sigma_{1}^{V}\left(M^{2}\right) d M^{2} M^{2}\left(M^{2}-s-i \epsilon\right)^{-1}
\end{aligned}
$$

Here $\gamma_{N N_{\rho}}$ is the coupling constant of $\rho$ to $\vec{u}_{f} i \tau \gamma_{\alpha} u_{i}$, just as $g_{N N \pi}$ is the coupling constant of $\pi$ to $\bar{u}_{f} i \tau \gamma_{5} u_{i}$. In this case, we have used an unsubtracted dispersion relation just for convenience.

Since the current is conserved, there is no renormalization and we have

$$
F_{1}{ }^{v}(0)=1,
$$

giving, in place of (2.7), the sum rule

$$
1=\gamma_{\rho N N} / \gamma_{\rho}+\int \sigma_{1}^{V}\left(M^{2}\right) d M^{2}
$$

If the dispersion relation is dominated at low $s$ by the $\rho$ term, then we obtain the analog of the GoldbergerTreiman formula:

$$
1 \approx \gamma_{\rho N N} / \gamma_{\rho}
$$

${ }^{9}$ G. F. Chew, University of California Radiation Laboratory Report No. UCRL-9289, 1960 (unpublished).

${ }^{10}$ M. Gell-Mann and I. Zachariasen, Phys. Rev. 124, 953 (1961). 
Now the same reasoning may be applied to the isovector electric form factor of another particle, for example the pion:

$$
\begin{aligned}
\left\langle\pi\left|\mathfrak{I}_{\alpha}\right| \pi\right\rangle= & {\left[i \boldsymbol{\phi}_{f}{ }^{*} \times \partial_{\alpha} \boldsymbol{\phi}_{i}-i \partial_{\alpha} \boldsymbol{\phi}_{f}{ }^{*} \times \boldsymbol{\phi}_{i}\right] F_{\pi}(s), } \\
F_{\pi}(s)= & \left(\gamma_{\rho \pi \pi} / \gamma_{\rho}\right) m_{\rho}{ }^{2}\left(m_{\rho}^{2}-s\right)^{-1} \\
& \quad+\int \sigma_{\pi}\left(M^{2}\right) d M^{2} M^{2}\left(M^{2}-s-i \epsilon\right)^{-1}
\end{aligned}
$$

and

$$
1=\gamma_{\rho \pi \pi} / \gamma_{\rho}+\int \sigma_{\pi}\left(M^{2}\right) d M^{2}
$$

If this dispersion relation, too, is dominated by the $\rho$ pole at low $s$, then we find

$$
1 \approx \gamma_{\rho \pi \pi} / \gamma_{\rho} \text {. }
$$

To the extent that the $\rho$ pole gives most of the sum rule in each case, we have $\rho$ coupled universally to the isotopic spins of nucleon, pion, etc., with coupling parameter $2 \gamma_{\rho}$. Such universality was postulated by Sakurai, ${ }^{11}$ within the framework of a special theory, in which $\rho$ is treated as an elementary vector meson described by a Yang-Mills field. It can be seen that whether or not such a field description is correct, the effective universality $\left(\gamma_{\rho \pi \pi} \approx \gamma_{\rho N N} \approx \gamma_{\rho K K}\right.$, etc. $)$ is an approximate rule the validity of which depends on the domination of (2.15), (2.19), etc., by the $\rho$ term.

The various coupling parameters $\gamma_{\rho \pi \pi}, \gamma_{\rho N N}$, etc., can be determined from the contribution of the $\rho$ "pole" to various scattering processes, for example $\pi+N \rightarrow \pi+N$. But the factors $\gamma_{\rho \pi \pi} / \gamma_{\rho}, \gamma_{\rho N N} / \gamma_{\rho}$, etc., can also be measured, using electromagnetic interactions. ${ }^{10}$

An approximate determination of $\gamma_{\rho N N} / \gamma_{\rho}$ was made by Hofstadter and Herman ${ }^{12}$ as follows The masses $M^{2}$ in the integral in Eq. (2.13) are taken to be effectively vary large, so that (2.13) becomes approximately

$$
\begin{aligned}
F_{1} V(s) \approx\left(\gamma_{N N_{\rho}} / \gamma_{\rho}\right) m_{\rho}{ }^{2}\left(m_{\rho}{ }^{2}-s\right)^{-1} & \\
& +1-\left(\gamma_{\rho N N} / \gamma_{\rho}\right) .
\end{aligned}
$$

Fitting the experimental data on $F_{1}^{V}(s)$ with such a formula and using $m_{\rho} \approx 750 \mathrm{Mev}$, we obtain $\gamma_{\rho N N} / \gamma_{\rho}$ $\approx 1.4$. (Hofstadter and Herman, with a smaller value of $m_{\rho}$, found 1.2.)

\section{EQUAL-TIME COMMUTATION RELATIONS}

The dispersion relations for the matrix elements of weak or electromagnetic currents are linear and homogeneous. For example, Eq. (2.6) may be thought of as an expression for the matrix element of $\mathbf{P}_{\alpha}$ between the vacuum and a nucleon-antinucleon pair state. On the right-hand side, the pole term contains the product of the matrix element of $\mathbf{P}_{\alpha}$ between the vacuum and a

${ }^{11} \mathrm{~J}$. J. Sakurai, Ann. Phys. 11, 1 (1960).

12 R. Hofstadter and R. Herman, Phys. Rev. Letters 6, 293 (1961). See also S. Bergia, A. Stanghellini, S. Fubini, and C. Villi, Phys. Rev. Letters 6, 367 (1961). one-pion state multiplied by the transition amplitude for the transition from $\pi$ to $N \bar{N}$ by means of the strong interactions. The weight function $\sigma_{\mathrm{ax}}\left(M^{2}\right)$ is just the sum of such products over many intermediate states (such as $3 \pi, 5 \pi$, etc.) with total mass $M$.

Now such linear, homogeneous equations may determine the dependence of the current matrix elements on variables such as $s$, but they cannot fix the scale of these matrix elements; constants like $-G_{A} / G$ cannot be calculated without further information. A field theory of the strong interactions, with explicit expressions for the currents, somehow contains more than these dispersion relations. In what follows, we shall extract some of this additional information in the form of equal-time commutation relations between components of the currents. Since these are nonlinear relations, they can help to fix the scale of each matrix element. Moreover, these relations may be the same for the lepton system and for the baryon-meson system, so that universality of strength of the weak interactions, for example, becomes meaningful. ${ }^{13}$

Let us begin our discussion of equal-time commutation relations with a familiar case - that of the isotopic spin I. Its components $I_{i}$ obey the well-known commutation relations

$$
\left[I_{i}, I_{j}\right]=i e_{i j k} I_{k} .
$$

In terms of the components $\Im_{i \alpha}$ of the isotopic spin current, we have

$$
I_{i}=-i \int \Im_{i 4} d^{3} x
$$

and the conservation law

tells us that

$$
\partial_{\alpha} \Im_{i \alpha}=0
$$

$$
\dot{I}_{i}=\int \partial_{\alpha} \Im_{i \alpha} d^{3} x=0
$$

at all times.

Now the commutator of $\Im_{i 4}(\mathbf{x}, t)$ and $\Im_{j 4}\left(\mathbf{x}^{\prime}, t\right)$ must vanish for $\mathbf{x} \neq \mathbf{x}^{\prime}$, in accorance with microcausality. (Note we have taken the times equal.) If the commutator is not more singular than a delta function, then (3.1) and (3.2) give us the relation

$$
\left[\Im_{i 4}(\mathbf{x}, t), \Im_{j 4}\left(\mathbf{x}^{\prime}, t\right)\right]=-i e_{i j k} \Im_{k 4}(\mathbf{x}, t) \delta\left(\mathbf{x}-\mathbf{x}^{\prime}\right),
$$

which can also be obtained in any simple field theory by explicit commutation. ${ }^{14}$

In discussing the various parts of the weak current $J_{\alpha}$, we shall have to deal with currents like $\mathbf{P}_{\boldsymbol{\alpha}}$ that are not

13 M. Gell-Mann, Proceedings of the 1960 Annual International Conference on High-Energy Physics at Rochester (Interscience Publishers, Inc., New York, 1960).

${ }^{14}$ In some cases explicit commutation may be ambiguous and misleading. For example, a superficial consideration of $\left[j_{i}(\mathbf{x}, t)\right.$, $\left.j_{4}\left(\mathbf{x}^{\prime}, t\right)\right]$ for $i=1,2,3$ may lead to the conclusion that the expression vanishes. Yet the vacuum expectation value of the commutator can be shown to be a nonzero quantity times $\partial_{i} \delta\left(\mathbf{x}-\mathbf{x}^{\prime}\right)$ and that result is confirmed by more careful calculation. See J. Schwinger, Phys. Rev. Letters 3, 296 (1959). 
conserved. ${ }^{15}$ Here, too, we may define a quantity analogous to $\mathbf{I}$ :

$$
D_{i}=-i \int P_{i 4} d^{3} x
$$

but $D_{i}$ is not independent of time:

$$
\dot{D}_{i}=\int \partial_{\alpha} P_{i \alpha} d^{3} x \neq 0 .
$$

For the moment, let us restrict our attention to the currents $\mathfrak{J}_{\boldsymbol{\alpha}}$ and $\mathbf{P}_{\boldsymbol{\alpha}}$ and the operators $\mathbf{I}$ and $\mathbf{D}(t)$. Since $\mathbf{D}$ is an isovector, we have the relations

$$
\left[I_{i}, D_{j}\right]=\left[D_{i}, I_{j}\right]=i e_{i j k} D_{k},
$$

but what is the commutator of two components of $\mathbf{D}$ ? Since $\mathbf{P}_{\alpha}$ is a physical quantity, so is $\mathbf{D}$ and the question is one with direct physical meaning. We shall give both a general and a specific answer.

In general, we may take the commutators of $D$ 's (divided by $i$ ), the components of $\mathbf{I}$ and $\mathbf{D}$, the commutators of all of these with one another (divided by $i$ ), etc., until we obtain a system of Hermitian operators that is closed under commutation. Any of these operators can be written as a linear combination of $N$ linearly independent Hermitian operators $R_{i}(t)$, where $N$ might be infinite, and where the commutator of any two $R_{i}$ is a linear combination of the $R_{i}$ :

$$
\left[R_{i}(t), R_{j}(t)\right]=i c_{i j k} R_{k}(t),
$$

with $c_{i j k}$ real. Such a system is called an algebra by the mathematicians. If we consider the set of infinitesimal unitary operators $1+i \epsilon R_{i}(t)$ and all possible products of these, we obtain an $N$-parameter continuous group of unitary transformations. We can refer to (3.9) as the algebra of the group. It is a physically meaningful statement to specify what group or what algebra is generated in this way by the currents $\mathfrak{\Im}_{\alpha}$ and $\mathbf{P}_{\alpha}$. Since a commutation relation like (3.9) is left invariant by a unitary transformation such as $\exp \left(-i t \int H d^{3} x\right)$, the numbers $c_{i j k}$ are independent of time.

A second mathematical statement is also in order, i.e., the specification of the transformation properties of the Hamiltonian density $H(\mathbf{x}, t)$ under the group or the algebra. Those $R_{i}$ for which $\left[R_{i}(t), H(\mathbf{x}, t)\right]=0$ are independent of time, but some of them, like $D_{i}$, do not commute with $H$. If all of the $R_{i}$ commuted with $H$, then $H$ would belong to the trivial one-dimensional representation of the group. In fact, $H$ behaves in a more complicated way. By commuting all of the $R_{i}(t)$ with $H(\mathbf{x}, t)$, we obtain a linear set of operators, containing $H$, that form a representation of the group; it may be broken up into the direct sum of irreducible representations. We want to know, then, what group is generated by $\mathbf{I}$ and $\mathbf{D}$ and to what irreducible repre-

\footnotetext{
${ }^{15}$ We assume that the vector weak current with $\Delta S=0$ is just a component of the isotopic spin current $\oiint_{\alpha}$ and thus conserved.
}

sentations of this group $H$ belongs. Suggested are specific answers to both questions.

Let us look at the vector and axial vector weak currents for the leptons. For the time being, we shall consider only $\nu$ and $e$, ignoring the muon. (In the same way, we shall, in this section, ignore strange particles, and consider only baryons and mesons with $S=0$.) The vector weak current $i \bar{\nu} \gamma_{\alpha} e$ and the axial current $i \bar{\nu} \gamma_{\alpha} \gamma_{5} e$ can be regarded formally as components of two "isotopic vector" currents for the leptons:

$$
\mathfrak{\Im}_{\alpha}{ }^{(l)}=i \bar{\xi}_{\tau \gamma_{\alpha} \xi / 2,} \quad \mathbf{P}_{\alpha}^{(l)}=i \bar{\xi}_{\tau \gamma_{\alpha} \gamma_{5} \xi / 2,}
$$

where $\xi$ stands for $(\nu, e)$. We can also form the mathematical analogs of $\mathbf{I}$ and $\mathbf{D}$ :

$$
\mathbf{I}^{(l)}=-i \int \mathfrak{I}_{\alpha}{ }^{(l)} d^{3} x, \quad \mathbf{D}^{(l)}=-i \int \mathbf{P}_{\alpha^{(l)}} d^{3} x .
$$

Now in this leptonic case we can easily compute the commutation rules of $\mathbf{I}^{(l)}$ and $\mathbf{D}^{(l)}$ :

$$
\begin{aligned}
{\left[I_{i}^{(l)}, I_{j}{ }^{(l)}\right] } & =i e_{i j k} I_{k}^{(l)}, \\
{\left[I_{i}^{(l)}, D_{j}{ }^{(l)}\right] } & =\left[D_{i}{ }^{(l)}, I_{j}{ }^{(l)}\right]=i e_{i j k} D_{k}{ }^{(l)}, \\
{\left[D_{i}{ }^{(l)}, D_{j}{ }^{(l)}\right] } & =i e_{i j k} I_{k}{ }^{(l)} .
\end{aligned}
$$

Another way to phrase these commutation rules is to put

$$
\begin{aligned}
\mathbf{I}^{(l)} & =\mathbf{L}_{\mathbf{+}}{ }^{(l)}+\mathbf{I}^{(l)}, \\
\mathbf{D}^{(l)} & =\mathbf{I}_{\mathbf{+}}{ }^{(l)}-\mathbf{I}^{(l)},
\end{aligned}
$$

and to notice that $\mathbf{I}_{+}{ }^{(l)}$ and $\mathbf{L}^{(l)}$ are two commuting angular momenta [essentially $\tau\left(1+\gamma_{5}\right) / 4$ and $\tau\left(1-\gamma_{5}\right) / 4$ ]. The weak current $i \bar{\nu} \gamma_{\alpha}\left(1+\gamma_{5}\right) e$ is just a component of the current of $\mathbf{L}_{+}{ }^{(l)}$.

We now suggest that the algebraic structure of $\mathbf{I}$ and D is exactly the same in the case of baryons and mesons. To (3.1) and (3.8), we add the rule ${ }^{16,17}$

$$
\left[D_{i}, D_{j}\right]=i e_{i j k} I_{k},
$$

which closes the system and makes $\mathbf{I}+\equiv(\mathbf{I}+\mathbf{D}) / 2$ and $\mathbf{I}^{-} \equiv(\mathbf{I}-\mathbf{D}) / 2$ two commuting angular momenta. Again, we make the weak current a component of the current of $\mathbf{I}^{+}$. Evidently the statement that $(\mathbf{I}+\mathbf{D}) / 2$ is an angular momentum and not some factor times an angular momentum, fixes the scale of the weak current. It makes universality of strength between baryons and leptons meaningful, and it specifies, together with the dispersion relations, the value of such constants as $-G_{A} / G$.

The simplest way to realize the algebraic structure under discussion in a field-theory model of baryons and mesons is to construct the currents $\mathfrak{I}_{\alpha}$ and $\mathbf{P}_{\alpha}$ out of $p$ and $n$ fields just as $\mathfrak{I}_{\alpha}{ }^{(l)}$ and $\mathbf{P}_{\boldsymbol{\alpha}}{ }^{(l)}$ are made out of $\nu$ and $e$ fields :

$$
\Im_{\alpha}=i \bar{N} \tau \gamma_{\alpha} N / 2, \quad \mathbf{P}_{\alpha}=i \bar{N} \tau \gamma_{\alpha} \gamma_{5} N / 2,
$$

${ }^{16}$ F. Gursey, Nuovo cimento 16, 230 (1960).

${ }^{17}$ M. Gell-Mann and M. Lévy, Nuovo cimento 16, 705 (1960). 
where $N$ means $(p, n)$. We then obtain not only the commutation rules (3.1), (3.8), and (3.14), but the stronger rule (3.5) and its analogs:

$$
\begin{aligned}
& {\left[\Im_{i 4}(\mathbf{x}, t), P_{j 4}\left(\mathbf{x}^{\prime}, t\right)\right]=-i e_{i j k} P_{k 4}(\mathbf{x}, t) \delta\left(\mathbf{x}-\mathbf{x}^{\prime}\right),} \\
& {\left[P_{i 4}(\mathbf{x}, t), P_{j 4}\left(\mathbf{x}^{\prime}, t\right)\right]=-i e_{i j k} \Im_{k 4}(\mathbf{x}, t) \delta\left(\mathbf{x}-\mathbf{x}^{\prime}\right) .}
\end{aligned}
$$

Next we want to use a field-theory model to suggest an answer to the second question-how $H$ behaves under the group or, what is the same thing, under the algebra consisting of $\mathbf{I}$ and $\mathbf{D}$ or of $\mathbf{I}^{+}$and $\mathbf{I}^{-}$. Since $\mathbf{I}^{+}$ and $\mathbf{I}^{-}$are two commuting angular momenta, any irreducible representation of the algebra is specified by a pair of total angular momentum quantum numbers: $i_{+}$for $\mathbf{I}^{+}$and $i_{-}$for $\mathbf{I}^{-}$. The total isotopic spin quantum number $I$ is associated with $\mathbf{I}^{+}+\mathbf{I}^{-}=\mathbf{I}$.

Now we want the vector weak current $\mathfrak{I}_{\alpha}$ to be the isotopic spin current and to be conserved. Thus $H$ must commute with $\mathbf{I}$; it transforms as an isoscalar, with $I=0$. In order to couple to zero, $i_{+}$and $i_{-}$must be equal. So $H$ can consist of terms with $\left(i_{+}, i_{-}\right)=(0,0)$, $\left(\frac{1}{2}, \frac{1}{2}\right),(1,1),\left(\frac{3}{2}, \frac{3}{2}\right)$, etc. Which of these are in fact present?

The simplest model in which the total isotopic current is given by just (3.15) is the Fermi-Yang ${ }^{18}$ model, in which the pion is a composite of nucleon and antinucleon. To write an explicit Lagrangian, it must be decided what form the binding interaction takes. Since a direct four-fermion coupling leads to unpleasant singularities, we whall use a massive neutral vector meson field $B^{0}$ coupled to the nucleon current, as proposed by Teller ${ }^{18}$ and Sakurai ${ }^{11}$; the exchange of a $B^{0}$ gives attraction between nucleon and antinucleon, permitting binding, and it also gives repulsion between nucleons, contributing to the "hard core." The model Lagrangian is then ${ }^{19}$

$$
\begin{aligned}
L=-\bar{N} \gamma_{\alpha} \partial_{\alpha} N-\left(\partial_{\alpha} B_{\beta}-\partial_{\beta} B_{\alpha}\right)^{2} / 4 \\
\quad-\mu_{0}^{2} B_{\alpha} B_{\alpha} / 2-i h_{0} B_{\alpha} \bar{N} \gamma_{\alpha} N-m_{0} \bar{N} N .
\end{aligned}
$$

If the mass term for the nucleon were absent, then both $\mathfrak{I}_{\boldsymbol{\alpha}}$ and $\mathbf{P}_{\boldsymbol{\alpha}}$ would be conserved; $\mathbf{I}$ and $\mathbf{D}$ would both commute with $L$ and with $H$. Thus,

$$
H=H(0,0)-u_{0}
$$

where $H(0,0)$ transforms according to $\left(i_{+}, i_{-}\right)=(0,0)$ and the noninvariant term $u_{0}$ is just $-m_{0} \bar{N} N$. To what representation does it belong?

It is easy to see that the field $B^{0}$ belongs to $(0,0)$, while $N_{L} \equiv\left(1+\gamma_{5}\right) N / 2$ belongs to $\left(\frac{1}{2}, 0\right)$ and $N_{R}$

18 E. Fermi and C. N. Yang, Phys. Rev. 76, 1739 (1949); E. Teller, Proceedings of the Sixth Annual Rochester Conference on High-Energy Nuclear Physics, 1956 (Interscience Publishers, Inc., New York, 1956).

${ }^{19}$ Conceivably a massive $B^{0}$ meson can be described by $(3.17)$ even with $\mu_{0}=0$. [J. Schwinger, lectures at Stanford University, summer, 1961 (unpublished) ]. In that case the noninvariant term in (3.17) is just equal to $\theta_{\alpha \alpha}$ and the traceless part of $\theta_{\alpha \beta}$ commutes with the group elements at equal times. In any case, whether $\mu_{0}$ is zero or not, the off-diagonal terms in $\theta_{\alpha \beta}$ commute with the group. $\equiv\left(1-\gamma_{5}\right) N / 2$ belongs to $\left(0, \frac{1}{2}\right)$. One can thus verify that all terms of (3.17) except the last belong to $(0,0)$, since $\bar{N} \gamma_{\alpha} N$ or $\bar{N} \gamma_{\alpha} \partial_{\alpha} N$ couples $\bar{N}_{L}$ to $N_{L}$ and $\bar{N}_{R}$ to $N_{R}$. But the Dirac matrix $\beta$, unlike $\beta \gamma_{\alpha}$, anticommutes with $\gamma_{5}$, so that the last term $-m_{0} \bar{N} N$ couples $\bar{N}_{L}$ to $N_{R}$ and $\bar{N}_{R}$ to $N_{L}$. Thus $u_{0}$ belongs to $\left(\frac{1}{2}, \frac{1}{2}\right)$. We have $H=H(0,0)+H\left(\frac{1}{2}, \frac{1}{2}\right)$.

There are four components to the representation $\left(\frac{1}{2}, \frac{1}{2}\right)$ to which $u_{0}=-H\left(\frac{1}{2}, \frac{1}{2}\right)$ belongs. By commuting $\mathbf{D}$ with $u_{0}$, we generate the other three easily and see that they are proportional to $-i \bar{N} \tau \gamma_{5} N$. In fact $\mathbf{D}$ acts like $\tau \gamma_{5} / 2$, I like $\tau / 2, u_{0}$ like $\beta$, and the other three components like $-i \beta \gamma_{5} \tau$. Denoting the three new components by $v_{i}$, we have

$$
\begin{array}{ll}
{\left[I_{i}, u_{0}\right]=0,} & {\left[D_{i}, u_{0}\right]=-i v_{i},} \\
{\left[I_{i}, v_{j}\right]=i e_{i j k} v_{k},} & {\left[D_{i}, v_{j}\right]=i \delta_{i j} u_{0} .}
\end{array}
$$

In the model, there are the even stronger relations for the densities

$$
\begin{aligned}
& {\left[\Im_{i 4}(\mathbf{x}, t), u_{0}\left(\mathbf{x}^{\prime}, t\right)\right]=0, } {\left[P_{i 4}(\mathbf{x}, t), u_{0}\left(\mathbf{x}^{\prime}, t\right)\right] } \\
&=-i v_{i}(\mathbf{x}, t) \delta\left(\mathbf{x}-\mathbf{x}^{\prime}\right), \text { etc. }
\end{aligned}
$$

The noninvariant term $u_{0}$ is what prevents the axial vector current from being conserved. Thus one can express the divergence $\partial_{\alpha} \mathbf{P}_{\alpha}$ of the current in terms of the commutator of $\mathbf{D}$ with $u_{0}$. The conditions for this relation to hold are treated in the appendix and are applicable to all models we discuss. We find simply

$$
\partial_{\alpha} \mathbf{P}_{\alpha}=-i[\mathbf{D}, H]=i\left[\mathbf{D}, u_{0}\right]=\mathbf{v},
$$

and, of course,

$$
\partial_{\alpha} \mathfrak{J}_{\alpha}=-i[\mathbf{I}, H]=0 \text {. }
$$

It is precisely the operator $\mathbf{v}$, then, that we used in a dispersion relation in order to obtain the GoldbergerTreiman relation in Sec. II. Acting on the vacuum, it leads mostly to the one-pion state, so that the pion is effectively coupled universally to the divergence of the axial vector current. Thus $\mathbf{v}$ is a sort of effective pion field operator for the Fermi-Yang theory, which has no explicit pion field.

If we insist on a model in which there is a field variable $\pi(x, t)$ then we must complicate the discussion. The total isotopic spin current is no longer given by just (3.15); there is a pion isotopic current term as well. In order to preserve the same algebraic structure of $\mathbf{I}$ and $\mathbf{D}$, one must then modify $\mathbf{P}_{\alpha}$ as well. Such a theory was described by Gell-Mann and Lévy, ${ }^{17}$ who called it the " $\sigma$-model". ${ }^{20}$ Along with the field $\pi$, we must introduce a scalar, isoscalar field $\sigma^{\prime}$ in such a way that $\pi, \sigma^{\prime}$ transform under the group like $\mathbf{v}, u_{0}$. Then, just as $\mathfrak{I}_{\alpha}$ has an additional term quadratic in $\boldsymbol{\tau}, \mathbf{P}_{\alpha}$ requires an additional term bilinear in $\boldsymbol{\pi}$ and $\sigma^{\prime}$.

As we shall see in the next section, the introduction of

${ }^{20}$ In the $\sigma$ model, explicit commutation of $u_{0}$ and $\mathbf{v}$ at equal times gives zero, while in the Fermi-Yang model this is not so if we take these results seriously, they give us definite physical distinctions among models. 
strange particles makes the group much larger. The term $u_{0}$ is then a member of a much larger representation, with eighteen components. Thus if a pion field is introduced, fifteen more components are needed as well. Such a theory is too complicated to be attractive; we shall therefore ignore it and concentrate on the simplest generalization of the Fermi-Yang model to strange particles, namely the symmetrical Sakata model.

\section{SYMMETRICAL SAKATA MODEL AND UNITARY SYMMETRY}

In the previous section, we proceeded inductively. We showed that starting from physical currents like $\mathfrak{T}_{\alpha}$ and $\mathbf{P}_{\alpha}$ we may construct a group and its algebra and that it is physically meaningful to specify the group and also the transformation properties of $H$ under the group. We chose the algebraic structure by analogy with the case of leptons and we saw that the simplest field theory model embodying the structure is just the Fermi-Yang model, in which $p$ and $n$ fields are treated just like the $\nu$ and $e$ fields for the leptons, except that they are given a mass and a strong "gluon" coupling. The transformation properties of $H$ were taken from the model $H$ consists, then, of an invariant part $H_{0,0}$ plus a term $\left(-u_{0}\right)$, where $u_{0}$ and a pseudoscalar isovector quantity $\mathbf{v}$ belong to the representation $\left(\frac{1}{2}, \frac{1}{2}\right)$ of the group. We then have the commutation rules (3.1), (3.8), (3.14), and (3.19). Microcausality with the assumption of commutators that are not too singular, or else direct inspection of the model, gives the stronger commutation rules (3.5), (3.16), and (3.20) for the densities. The model also gives specific equal-time commutation rules for $u_{0}$ and $\mathbf{v}$, which we did not list. All of these properties can be abstracted from the model and considered on their own merits as proposed relations among the currents and the Hamiltonian density.

Now, to argue deductively, we want to include the strange particles and all parts of the weak current $J_{\alpha}$ and the electromagnetic current $j_{\alpha}$. We generalize the Fermi-Yang description to obtain the symmetrical Sakata model and abstract from it as many physically meaningful relations as possible.

It has long been recognized that the qualitative properties of baryons and mesons could be understood in terms of the Sakata model, ${ }^{21}$ in which all strongly interacting particles are made out of $N, \Lambda, \bar{N}$, and $\bar{\Lambda}$ (or at least out of basic fields with the same quantum numbers as these particles).

We write the Lagrangian density for the Sakata model as a generalization of (3.17):

$$
\begin{array}{r}
L=-\bar{p} \gamma_{\alpha} p-\bar{n} \gamma_{\alpha} \partial_{\alpha} \bar{n}-\bar{\Lambda} \gamma_{\alpha} \partial_{\alpha} \Lambda-\frac{1}{4}\left(\partial_{\alpha} B_{\beta}-\partial_{\beta} B_{\alpha}\right)^{2} \\
-\frac{1}{2} \mu_{0}^{2} B_{\alpha} B_{\alpha}-i h_{0}\left(\bar{p} \gamma_{\alpha} p+\bar{n} \gamma_{\alpha} n+\bar{\Lambda} \gamma_{\alpha} \Lambda\right) B_{\alpha} \\
-m_{0 N}(\bar{n} n+\bar{p} p)-m_{0 \Lambda} \bar{\Lambda} \Lambda .
\end{array}
$$

According to this picture, the baryons present a

\footnotetext{
${ }^{21}$ S. Sakata, Progr. Theoret. Phys. (Kyoto) 16, 686 (1956).
}

striking parallel with the leptons, ${ }^{22}$ for which we write the Lagrangian density

$L_{l}=-\bar{\nu} \gamma_{\alpha} \nu-\bar{e} \gamma_{\alpha} \partial_{\alpha} e-\bar{\mu} \gamma_{\alpha} \partial_{\alpha} \mu-0 \cdot(\bar{\nu} \nu+\bar{e} e)-m_{\mu} \bar{\mu} \mu$,

if we turn off the electromagnetic and weak couplings, along with the $\nu$-e mass difference. Here it is assumed there is only one kind of neutrino.

The only real difference between baryons and leptons in (4.1) and (4.2), respectively, is that the baryons are coupled, through the baryon current, to the field $B$. It is tempting to suppose that the weak current of the strongly interacting particles is just the expression.

$$
i \bar{p} \gamma_{\alpha}\left(1+\gamma_{5}\right) n+i \bar{p} \gamma_{\alpha}\left(1+\gamma_{5}\right) \Lambda
$$

analogous to Eq. (1.2) for the leptonic weak current $J_{\alpha}{ }^{(l)}$. Now (4.3) is certainly a reasonable expression, qualitatively, for weak currents of baryons and mesons. As Okun has emphasized, ${ }^{23}$ the following properties of the weak interactions, often introduced as postulates, are derivable from (1.1), (1.2), (4.1), and (4.3):

(a) The conserved vector current. ${ }^{24}$ In the model under discussion, as in that of Fermi and Yang, $i \bar{p} \gamma_{\alpha} n$ is a component of the total isotopic spin current.

(b) The rules $|\Delta S|=1, \Delta S / \Delta Q=+1$, and $|\Delta \mathbf{I}|=\frac{1}{2}$ for the leptonic decays of strange particles. ${ }^{25}$

(c) The invariance under GP of the $\Delta S=0$ weak current. ${ }^{26}$

(d) The rules $|\Delta S|=1,|\Delta \mathbf{I}|=\frac{1}{2}$ or $\frac{3}{2}$ in the nonleptonic decays of strange particles; along with $|\Delta S|$ $=1$, we have the absence of a large $K_{1}{ }^{0}-K_{2}{ }^{0}$ mass difference.

The quantitative facts that the effective coupling constants for $|\Delta S|=1$ leptonic decays are smaller than those for $|\Delta S|=0$ leptonic decays and that in nonleptonic decays of strange particles the $|\Delta \mathbf{I}|=\frac{1}{2}$ amplitude greatly predominates over the $|\Delta \mathbf{I}|=\frac{3}{2}$ amplitude are not explained in any fundamental way. ${ }^{27}$

${ }^{22}$ A. Gamba, R. E. Marshak, and S. Okubo, Proc. Natl. Acad. Sci. U. S. 45, 881 (1959).

${ }_{23}$ L. Okun, Ann. Rev. Nuclear Sci. 9, 61 (1959).

${ }^{24}$ R. P. Feynman and M. Gell-Mann, Phys. Rev. 109, 193 (1958). See also S. S. Gershtein and J. B. Zeldovich, Soviet Phys.-JETP 2, 576 (1957).

${ }_{25}^{25}$ M. Gell-Mann, Proceedings of the Sixth Annual Rochester Conference on High-Energy Nuclear Physics, 1956 (Interscience Publishers, Inc., New York, 1956). These rules were in fact suggested on the basis of the idea that $N$ and $\Lambda$ are fundamental. Should the rules prove too restrictive (for example should $\Delta S / \Delta Q$ $=+1$ be violated), then we would try a larger group; in the language of the field-theoretic model, we would assume more fundamental fields. For a discussion of possible larger groups, see M. Gell-Mann and S. Glashow, Ann. Phys. 15, 437 (1961) and S. Coleman and S. Glashow (to be published).

${ }^{26}$ S. Weinberg, Phys. Rev. 112, 1375 (1958).

${ }^{27} \mathrm{~A}$ possible dynamical explanation of the predominance of $|\Delta \mathbf{I}|=\frac{1}{2}$ is being investigated by Nishijima (private communication). For example, consider the decay $\Lambda \rightarrow N+\pi$. A dispersion relation without subtractions is written for the matrix element of $J_{\alpha}^{\dagger} J_{\alpha}$ between the vacuum and a state containing $N+\bar{\Lambda}+\pi$. The parity-violating part leads to intermediate pseudoscalar states with $S=+1$ and with $|\Delta \mathbf{I}|=\frac{1}{2}$ or $\frac{3}{2}$. In the case of $|\Delta \mathbf{I}|=\frac{1}{2}$, there is an intermediate $K$ particle, which may give a large contribution, swamping the term with $|\Delta \mathbf{I}|=\frac{3}{2}$, which has no one-meson state. For the same argument to apply to the parity-conserving part, we need the $K^{\prime}$ meson of Table III. 
TABLE I. A set of matrices $\lambda_{i}$.

\begin{tabular}{lll}
$\lambda_{1}=\left(\begin{array}{lll}0 & 1 & 0 \\
1 & 0 & 0 \\
0 & 0 & 0\end{array}\right)$ & $\lambda_{2}=\left(\begin{array}{rrr}0 & -i & 0 \\
i & 0 & 0 \\
0 & 0 & 0\end{array}\right)$ & $\lambda_{3}=\left(\begin{array}{rrr}1 & 0 & 0 \\
0 & -1 & 0 \\
0 & 0 & 0\end{array}\right)$ \\
$\lambda_{4}=\left(\begin{array}{lll}0 & 0 & 1 \\
0 & 0 & 0 \\
1 & 0 & 0\end{array}\right)$ & $\lambda_{5}=\left(\begin{array}{rrr}0 & 0 & -i \\
0 & 0 & 0 \\
i & 0 & 0\end{array}\right)$ & $\lambda_{6}=\left(\begin{array}{lll}0 & 0 & 0 \\
0 & 0 & 1 \\
0 & 1 & 0\end{array}\right)$ \\
$\lambda_{7}=\left(\begin{array}{rrr}0 & 0 & 0 \\
0 & 0 & -i \\
0 & i & 0\end{array}\right)$ & $\lambda_{8}=\left(\begin{array}{rrr}1 / \sqrt{3} & 0 & 0 \\
0 & 1 / \sqrt{3} & 0 \\
0 & 0 & -2 / \sqrt{3}\end{array}\right)$ \\
\hline
\end{tabular}

The electromagnetic properties of baryons and leptons are not exactly parallel in the Sakata model. The electric current (divided by $e$ ), which are denoted by $j_{\alpha}$, is given by

$$
i \bar{p} \gamma_{\alpha} p
$$

for the baryons and mesons and by

for the leptons.

$$
-i\left(\bar{e} \gamma_{\alpha} e+\bar{\mu} \gamma_{\alpha} \mu\right)
$$

Now, we return to the Lagrangian (4.1) and separate it into three parts:

$$
L=\bar{L}+L^{\prime}+L^{\prime \prime},
$$

where $\bar{L}$ stands for everything except the baryon mass terms, while $L^{\prime}$ and $L^{\prime \prime}$ are given by the expressions

$$
\begin{aligned}
L^{\prime} & =\left(2 m_{0 N}+m_{0 \Lambda}\right)(\bar{N} N+\bar{\Lambda} \Lambda) / 3, \\
L^{\prime \prime} & =\left(m_{0 N}-m_{0 \Lambda}\right)(\bar{N} N-2 \bar{\Lambda} \Lambda) / 3 .
\end{aligned}
$$

If we now consider the Lagrangian with the masssplitting term $L^{\prime \prime}$ omitted, we have a theory that is completely symmetrical in $p, n$, and $\Lambda$. We may perform any unitary linear transformation (with constant coefficients) on these three fields and leave $\bar{L}+L^{\prime}$ invariant. Thus in the absence of the mass-splitting term $L^{\prime \prime}$ the theory is invariant under the three-dimensional unitary group $U(3)$; we shall refer to this situation as "unitary symmetry."

If we now turn on the mass-splitting, the symmetry is reduced. The only allowed unitary transformations are those involving $n$ and $p$ alone or $\Lambda$ alone. The group becomes $U(2) \times U(1)$, which corresponds, as we shall see, to the conservation if isotopic spin, strangeness, and baryon number.

For simplicity, let us return briefly to the simpler case in which there is no $\Lambda$. The symmetry group is then just $U(2)$, the set of unitary transformations on $n$ and $p$. We can factor each unitary transformation uniquely into one which multiplies both fields by the same phase factor and one (with determinant unity) which leaves invariant the product of the phase factors of $p$ and $n$. Invariance under the first kind of transformation corresponds to conservation of nucleons $n$ and $p$; it may be considered separately from invariance under the class of transformations of the second kind [called by mathematicians the unitary unimodular group $S U(2)$ in two dimensions]. In mathematical language, we can factor $U(2)$ into $U(1) \times S U(2)$.

Each transformation of the first kind can be written as a matrix $1 \exp i \phi$, where 1 is the unit $2 \times 2$ matrix. The infinitesimal transformation is $1+i 1 \delta \phi$, and so the unit matrix is the infinitesimal generator of these transformations. Those of the second kind are generated in the same way by the three independent traceless $2 \times 2$ matrices, which may be taken to be the Pauli isotopic spin matrices $\tau_{1}, \tau_{2}$, and $\tau_{3}$. We thus have

$$
N \rightarrow\left(1+i \sum_{k=1}^{\mathbf{3}} \delta \theta_{k} \tau_{k} / 2\right) N
$$

as the general infinitesimal transformation of the second kind. Symmetry under all the transformations of the second kind is the same as symmetry under isotopic spin rotations. The whole formalism of isotopic spin theory can then be constructed by considering the transformation properties of the doublet or spinor $(p, n)$ and of more complicated objects that transform like combinations of two or more such nucleons (or antinucleons).

The Pauli matrices $\tau_{k}$ are Hermitian and obey the rules

$$
\begin{aligned}
\operatorname{Tr} \tau_{i} \tau_{j} & =2 \delta_{i j}, \\
{\left[\tau_{i}, \tau_{j}\right] } & =2 i e_{i j k} \tau_{k}, \\
\left\{\tau_{i}, \tau_{j}\right\} & =2 \delta_{i j} 1 .
\end{aligned}
$$

The invariance under the group $S U(2)$ of isotopic spin rotations corresponds to conservation of the isotopic spin current

$$
\mathfrak{\Im}_{\alpha}=i \bar{N} \tau \gamma_{\alpha} N / 2,
$$

while the invariance under transformations of the first kind corresponds to conservation of the nucleon current $i \bar{N} \gamma_{\alpha} N / 2=n_{\alpha}$.

Defining the total isotopic spin I as in (3.2), we obtain for $I_{i}$ the commutation rules (3.1), which are the same as those for $\tau_{i} / 2$. Likewise the nucleon number is defined as $-i \int n_{4} d^{3} x$ and commutes with $\mathbf{I}$.

We now generalize the idea of isotopic spin by including the third field $\Lambda$. Again we factor the unitary transformations on baryons into those which are generated by the $3 \times 3$ unit matrix 1 (and which correspond to baryon conservation) and those which are generated by the eight independent traceless $3 \times 3$ matrices [and which form the unitary unimodular group $S U(3)$ in three dimensions]. We may construct a typical set of eight such matrices by analogy with the $2 \times 2$ matrices of Pauli. We call then $\lambda_{1} \cdots \lambda_{8}$ and list them in Table $\mathrm{I}$. They are Hermitian and have the properties

$$
\begin{aligned}
& \operatorname{Tr} \lambda_{i} \lambda_{j}=2 \delta_{i j}, \\
& {\left[\lambda_{i}, \lambda_{j}\right]=2 i f_{i j k} \lambda_{k},} \\
& \left\{\lambda_{i}, \lambda_{j}\right\}=2 d_{i j k} \lambda_{k}+\frac{4}{3} \delta_{i j} 1,
\end{aligned}
$$

where $f_{i j k}$ is real and totally antisymmetric like the 
TABLE II. Nonzero elements of $f_{i j k}$ and $d_{i j k}$. The $f_{i j k}$ are odd under permutations of any two indices while the $d_{i j k}$ are even.

\begin{tabular}{cccc}
\hline \hline$j k$ & $f_{i j k}$ & $i j k$ & $d_{i j k}$ \\
\hline 123 & 1 & 118 & $1 / \sqrt{3}$ \\
147 & $1 / 2$ & 146 & $1 / 2$ \\
156 & $-1 / 2$ & 157 & $1 / 2$ \\
246 & $1 / 2$ & 228 & $1 / \sqrt{3}$ \\
257 & $1 / 2$ & 247 & $-1 / 2$ \\
345 & $1 / 2$ & 256 & $1 / 2$ \\
367 & $-1 / 2$ & 338 & $1 / \sqrt{3}$ \\
458 & $\sqrt{3} / 2$ & 344 & $1 / 2$ \\
678 & $\sqrt{3} / 2$ & 355 & $1 / 2$ \\
$\ldots$ & $\cdots$ & 366 & $-1 / 2$ \\
$\ldots$ & $\cdots$ & 377 & $-1 / 2$ \\
$\ldots$ & $\cdots$ & 448 & $-1 /(2 \sqrt{3})$ \\
$\ldots$ & $\cdots$ & 558 & $-1 /(2 \sqrt{3})$ \\
$\ldots$ & $\cdots$ & 668 & $-1 /(2 \sqrt{3})$ \\
$\ldots$ & $\cdots$ & 778 & $-1 /(2 \sqrt{3})$ \\
\hline & $\cdots$ & 888 & $-1 / \sqrt{3}$ \\
\hline
\end{tabular}

Kronecker symbol $e_{i j k}$ of Eq. (4.9), while $d_{i j k}$ is real and totally symmetric. These properties follow from the equations

$$
\begin{aligned}
& \operatorname{Tr} \lambda_{k}\left[\lambda_{i}, \lambda_{j}\right]=4 i f_{i j k}, \\
& \operatorname{Tr} \lambda_{k}\left\{\lambda_{i}, \lambda_{j}\right\}=4 d_{i j k},
\end{aligned}
$$

derived from (4.10).

The nonzero elements of $f_{i j k}$ and $d_{i j k}$ are given in Table II for our choice of $\lambda_{i}$. Even and odd permutations of the listed indices correspond to multiplication of $f_{i j k}$ by \pm 1 , respectively, and of $d_{i j k}$ by +1 .

The general infinitesimal transformation of the second kind on the three basic baryons $b$ is, of course,

$$
b \rightarrow\left(1+i \sum_{i=1}^{8} \delta \theta_{i} \lambda_{i} / 2\right) b
$$

by analogy with (4.8). Together with conservation of baryons, invariance under these transformations corresponds to complete "unitary symmetry" of the three baryons. We have factored $U(3)$ into $U(1) \times S U(3)$.

The invariance under transformations of the first kind gives us conservation of the baryon current

$$
i \bar{b} \gamma_{\alpha} b=i \bar{n} \gamma_{\alpha} n+i \bar{p} \gamma_{\alpha} p+i \bar{\Lambda} \gamma_{\alpha} \Lambda,
$$

while invariance under the second class of transformations would give us conservation of the eight-component "unitary spin" current

$$
\mathfrak{F}_{i \alpha}=i \bar{b} \lambda_{i} \gamma_{\alpha} b / 2 \quad(i=1, \cdots, 8) .
$$

Now in fact $L^{\prime \prime}$ is not zero and so not all the components of $\mathscr{F}_{i \alpha}$ are actually conserved. This does not prevent us from defining $\widetilde{F}_{i \alpha}$ as in (4.14), nor does it affect the commutation rules of the unitary spin density. The total unitary spin $F_{i}$ is defined by the relation

$$
F_{i}=-i \int \mathcal{F}_{i 4} d^{3} x
$$

at any time and at equal times the commutation rules for $F_{i}$ follow those for $\lambda_{i} / 2$

$$
\left[F_{i}, F_{j}\right]=i f_{i j k} F_{k} \text {. }
$$

The baryon number, of course, commutes with all components $F_{i}$.

It will be noticed that $\lambda_{1}, \lambda_{2}$, and $\lambda_{3}$ agree with $\tau_{1}, \tau_{2}$, and $\tau_{3}$ for $p$ and $n$ and have no matrix elements for $\Lambda$. Thus the first three components of the unitary spin are just the components of the isotopic spin. The matrix $\lambda_{8}$ is diagonal in our representation and has one eigenvalue for the nucleon and another for the $\Lambda$. Thus $F_{8}$ is just a linear combination of strangeness and baryon number. It commutes with the isotopic spin.

The matrices $\lambda_{4}, \lambda_{5}, \lambda_{6}$, and $\lambda_{7}$ connect the nucleon and $\Lambda$. We see that the components $F_{4}, F_{5}, F_{6}$, and $F_{7}$ of the unitary spin change strangeness by one unit and isotopic spin by a half unit. When the mass-splitting term $L$ " is "turned on," it is these components that are no longer conserved, while the conservation of $F_{1}, F_{2}$, $F_{3}, F_{8}$, and baryon number remains valid.

\section{VECTOR AND AXIAL VECTOR CURRENTS}

We may unify the mathematical treatment of the baryon current and the unitary spin current if we define a ninth $3 \times 3$ matrix

$$
\lambda_{0}=\left(\frac{2}{3}\right)^{\frac{1}{2}} 1
$$

so that the nine matrices $\lambda_{i}$ obey the rules

$$
\begin{array}{ll}
{\left[\lambda_{i}, \lambda_{j}\right]=2 i f_{i j k} \lambda_{k}} & (i=0, \cdots, 8), \\
\left\{\lambda_{i}, \lambda_{j}\right\}=2 d_{i j k} \lambda_{k} & (i=0, \cdots, 8), \\
\operatorname{Tr} \lambda_{i} \lambda_{j}=2 \delta_{i j} & (i=0, \cdots, 8) .
\end{array}
$$

Here, $f_{i j k}$ is defined as before, except that it vanishes when any index is zero; $d_{i j k}$ is also defined as before, except that it has additional nonzero matrix elements equal to $\left(\frac{2}{3}\right)^{\frac{1}{2}}$ whenever any index is zero and the other two indices are equal. The baryon current is now $\left(\frac{3}{2}\right)^{\frac{1}{2}} \mathcal{F}_{0 \alpha}$.

The definitions (4.15) and the equal-time commutation relations (4.16) now hold for $i=0, \cdots, 8$. Moreover, there are the equal-time commutation relations

$$
\left[\mathfrak{F}_{i 4}(\mathbf{x}, t), \mathfrak{F}_{j 4}\left(\mathbf{x}^{\prime}, t\right)\right]=-i f_{i j k} \mathfrak{F}_{k 4}(\mathbf{x}, t) \delta\left(\mathbf{x}-\mathbf{x}^{\prime}\right)
$$

for the densities.

The electric current $j_{\alpha}$ is then

$$
j_{\alpha}=\left(\sqrt{2} \mathscr{F}_{0 \alpha}+\mathfrak{F}_{8 \alpha}+\sqrt{3} \mathfrak{F}_{3 \alpha}\right) / 2 \sqrt{3},
$$

while the vector weak current is

$$
\mathfrak{F}_{1 \alpha}+i \mathfrak{F}_{2 \alpha}+\mathfrak{F}_{4 \alpha}+i \mathfrak{F}_{5 \alpha} .
$$

We now wish to set up the same formalism for the axial vector currents. We recall that the presence of the symmetry-breaking term $L^{\prime \prime}$ did not prevent us from defining the $\mathcal{F}_{i \alpha}$ and obtaining the commutation rules (5.3) characteristic of the unitary symmetry group $U(3)$.

In the same way, we now remark that if both $L^{\prime \prime}$ 
and $L^{\prime}$ are "turned off," we have invariance under the infinitesimal unitary transformations

$$
b \rightarrow\left(1+i \sum_{i=0}^{8} \delta \psi_{i} \gamma_{5} \lambda_{i} / 2\right) b,
$$

as well as the infinitesimal transformations

$$
b \rightarrow\left(1+i \sum_{i=0}^{8} \delta \theta_{i} \lambda_{i} / 2\right) b
$$

we have used before. ${ }^{28}$ Thus the axial vector currents

$$
\mathcal{F}_{i \alpha}{ }^{5}=i \bar{b} \lambda_{i} \gamma_{5} b / 2
$$

would be conserved if both $L^{\prime}$ and $L^{\prime \prime}$ were absent. Even in the presence of these terms, we have the commutation rules and

$$
\left[\mathfrak{F}_{i 4}{ }^{5}(\mathbf{x}, t), \mathfrak{F}_{j 4}\left(\mathbf{x}^{\prime}, t\right)\right]=-i f_{i j k} \mathfrak{F}_{k 4}{ }^{5}(\mathbf{x}, t) \delta\left(\mathbf{x}-\mathbf{x}^{\prime}\right)
$$

$$
\left[\mathfrak{F}_{i 4}{ }^{5}(\mathbf{x}, t), \mathfrak{F}_{j 4}{ }^{5}\left(\mathbf{x}^{\prime}, t\right)\right]=-i f_{i j k} \mathfrak{F}_{k 4}(\mathbf{x}, t) \delta\left(\mathbf{x}-\mathbf{x}^{\prime}\right)
$$

at equal times, We may use the definition

$$
F_{i}{ }^{5}(t) \equiv-i \int \mathcal{F}_{i 4}{ }^{5} d^{3} x
$$

along with (4.15)

Just as we put $\mathbf{I}=\mathbf{I}_{+}+\mathbf{L}$ and $\mathbf{D}=\mathbf{L}_{+}-\mathbf{I}$ in the discussion following Eq. (3.16), so we now write

$$
\begin{aligned}
F_{i}(t) & =F_{i}{ }^{+}(t)+F_{i}-(t), \\
F_{i}{ }^{5}(t) & =F_{i}{ }^{+}(t)-F_{i}{ }^{-}(t),
\end{aligned}
$$

and it is seen that $F_{i}^{+}$and $F_{i}^{-}$separately obey the commutation rules

$$
\left[F_{i}^{ \pm}, F_{j}^{ \pm}\right]=i f_{i j k} F_{k}^{ \pm}
$$

while they commute with each other:

$$
\left[F_{i}^{ \pm}, F_{j}^{ \pm}\right]=0
$$

Thus we are now dealing with the group $U(3)$ taken twice: $U(3) \times U(3)$. Factoring each $U(3)$ into $U(1)$ $\times S U(3)$, we have ${ }^{29} U(1) \times U(1) \times S U(3) \times S U(3)$. Thus we have defined a left- and a right-handed number and a left- and right-handed unitary spin.

The situation is just as in Sec. III, where we defined a left- and a right-handed isotopic spin and we could have defined a left- and a right-handed nucleon number.

The left- and right-handed quantities are connected

${ }^{28}$ Actually the Lagrangian (4.1) without the nucleon mass terms is invariant under a larger continuous group of transformations than the one $[U(3) \times U(3)]$ that we treat here. For example, there are infinitesimal transformations in which the baryon fields $b$ acquire small terms in $\bar{b}$. Invariance under these is associated with the conservation of currents carrying baryon number 2 . The author wishes to thank Professor W. Thirring for a discussion of these additional symmetries and of conformal transformations, which give still more symmetry.

${ }^{29}$ The groups $U(1), S U(3)$, and $S U(2)$ cannot be further factored in this fashion. They are called simple. to each other by the parity operation $P$ :

$$
P F_{i}{ }^{ \pm} P^{-1}=F_{i}^{\mp} \text {. }
$$

Now that we have constructed the mathematical apparatus of the group $U(3) \times U(3)$ and its algebra, we may inquire how the Hamiltonian density $H$ behaves under the group, i.e., under commutation with the algebra.

In the model, there is, corresponding to (4.6), the formula

$$
H=\bar{H}-L^{\prime}-L^{\prime \prime},
$$

where $\bar{H}$ is the Hamiltonian density derived from the Lagrangian density $\bar{L}$ and is completely invariant under the group. Instead of defining $u_{0}$ as in Sec. III, let us put

$$
u_{0}=L^{\prime} \propto \bar{b} \lambda_{0} b .
$$

We can easily see that by commutation of $u_{0}$ with $F_{i}$ and $F_{i}{ }^{5}(i=0, \cdots, 8)$ at equal times we obtain a set of eighteen quantities:

$$
\begin{aligned}
& u_{i} \propto \bar{b} \lambda_{i} b, \\
& v_{i} \propto-i \bar{b} \lambda_{i} \gamma_{5} b .
\end{aligned}
$$

In fact $F_{i}$ acts like $\lambda_{i} / 2, F_{i}{ }^{5}$ like $\lambda_{i} \gamma_{5} / 2, u_{i}$ like $\beta \lambda_{i}$, and $v_{i}$ like $-i \beta \gamma_{5} \lambda_{i}$. Thus we have at equal times ${ }^{30}$

$$
\begin{aligned}
{\left[F_{i}, u_{j}\right] } & =i f_{i j k} u_{k}, \\
{\left[F_{i}, v_{j}\right] } & =i f_{i j k} v_{k}, \\
{\left[F_{i}{ }^{5}, u_{j}\right] } & =-i d_{i j k} v_{k}, \\
{\left[F_{i}{ }^{5}, v_{j}\right] } & =i d_{i j k} u_{k},
\end{aligned}
$$

and the stronger relations

$$
\left[\mathfrak{F}_{i 4}(\mathbf{x}, t), u_{j}\left(\mathbf{x}^{\prime}, t\right)\right]=-f_{i j k} u_{k}(\mathbf{x}, t) \delta\left(\mathbf{x}-\mathbf{x}^{\prime}\right) \text {, etc. }
$$

for the densities. All indices run from 0 to 8 .

Note that we can now express not only $L^{\prime}$ (which is defined to be $u_{0}$ ) but $L^{\prime \prime}$ as well, since by (4.7) it is proportional to $u_{8}$. We have, then,

$$
H=\bar{H}=u_{0}-c u_{8},
$$

where $c$ is of the order $\left(m_{0 N}-m_{0 \Lambda}\right) / m_{0 N}$ in the model.

We may now make a series of abstractions from the model. First, we suppose that currents $\mathscr{F}_{i \alpha}$ and $\mathscr{F}_{i \alpha}{ }^{5}$ are defined, with commutation rules (5.3), (5.9), and (5.10), and with the weak current given by the analog of $(5.5)^{31}$ :

$$
\begin{aligned}
J_{\alpha}=\mathfrak{F}_{1 \alpha}+\mathcal{F}_{1 \alpha}{ }^{5}+i \mathfrak{F}_{2 \alpha} & +i F_{2 \alpha}{ }^{5} \\
& +\mathcal{F}_{4 \alpha}+\mathscr{F}_{4 \alpha}{ }^{5}+i \mathfrak{F}_{5 \alpha}+i F_{5 \alpha}{ }^{5},
\end{aligned}
$$

${ }^{30}$ Note that even if we use just $F_{i}$ and $F_{i}{ }^{5}$ for $i=1, \cdots, 8$, or $S U(3) \times S U(3)$ only, we still generate all eighteen $u$ 's and $v$ 's. IIn the two-dimensional case described in Sec. III the situation is different. Using $S U(2) \times S U(2)$, we generate from $u_{0}$ only itself and $v_{1}, v_{2}, v_{3}$; if we then bring in $F_{0}^{5}$ as well, we obtain three more $u$ 's and one more $v$.] This remark is interesting because the group that gives currents known to be physically interesting is just $U(1) \times S U(3) \times S U(3)$; there is no known physical coupling to $\mathscr{F}_{0 \alpha^{5}}$, the axial vector baryon current.

${ }^{31}$ Note that the total weak current, whether for baryons and mesons or for leptons, is just a component of the current of an angular momentum. See reference 13 . 
while the electric current is given by (5.4). Next, we may take the Hamiltonian density to be of the form (5.21), with $\bar{H}$ invariant and $u_{i}$ and $v_{i}$ transforming as in (5.20). Then, if the theory is of the type described in Appendix A, we can calculate the divergences of the currents in terms of the equal-time commutators

$$
\begin{aligned}
\partial_{\alpha} F_{i \alpha} & =i\left[F_{i}, u_{0}\right]+i c\left[F_{i}, u_{8}\right], \\
\partial_{\alpha} \mathfrak{F}_{i \alpha}{ }^{5} & =i\left[F_{i}{ }^{5}, u_{0}\right]+i c\left[F_{i}{ }^{5}, u_{8}\right],
\end{aligned}
$$

or, explicitly,

$$
\begin{aligned}
\partial_{\alpha} F_{i \alpha} & =0, \quad(i=0,1,2,3,8) \\
\partial_{\alpha} F_{4 \alpha} & =\left(\frac{3}{2}\right)^{\frac{1}{2}} u_{5}, \text { etc. } \\
\partial_{\alpha} F_{0 \alpha} & =\left(\frac{2}{3}\right)^{\frac{1}{2}} v_{0}+\left(\frac{2}{3}\right)^{\frac{1}{2}} c v_{\alpha}, \\
\partial_{\alpha} F_{1 \alpha} & =\left[\left(\frac{2}{3}\right)^{\frac{1}{2}}+\left(\frac{1}{3}\right)^{\frac{1}{2}} c\right] v_{1}, \text { etc. } \\
\partial_{\alpha} F_{4 \alpha} & =\left[\left(\frac{2}{3}\right)^{\frac{1}{2}}-\left(\frac{1}{12}\right)^{\frac{1}{2}} c\right] v_{4}, \text { etc. } \\
\partial_{\alpha} F_{8 \alpha} & =\left[\left(\frac{2}{3}\right)^{\frac{1}{2}}-\left(\frac{1}{3}\right)^{\frac{1}{2}} c\right] v_{8}+\left(\frac{2}{3}\right)^{\frac{1}{2}} c v_{0} .
\end{aligned}
$$

Finally, if we taken the model really seriously, we may abstract the equal-time commutation relations of the $u_{i}$ and $v_{i}$ as obtained by explicit commutation in the model.

The relations of Sec. III are all included in those of this section, except that what was called $u_{0}$ there is now called $u_{0}+c u_{8}$ and what was called $v_{i}$ is now called $\left[\left(\frac{2}{3}\right)^{\frac{1}{3}}+\left(\frac{1}{3}\right)^{\frac{1}{2}} c\right] v_{i}$ for $i=1,2,3$.

All of the relations used here are supposed to be exact and are not affected by the symmetry-breaking character of the non-invariant term in the Hamiltonian. In the next section, we discuss what happens if $c$ can be regarded as small in any sense. We may then expect to see some trace of the symmetry under $U(3)$ that would obtain if $c$ were 0 and $L^{\prime \prime}$ disappeared. In this limit, $N$ and $\Lambda$ are degenerate, and all the components $F_{i}$ of the unitary spin are conserved. The higher symmetry would show up particularly through the existence of degenerate baryon and meson supermultiplets, which break up into ordinary isotopic multiplets when $L^{\prime \prime}$ is turned on. These supermultiplets have been discussed previously for baryons and pseudoscalar mesons ${ }^{4,6}$ and then for vector mesons. . $^{32-34}$

We shall not discuss the case in which both $L^{\prime}$ and $L^{\prime \prime}$ are turned off; that is the situation, still more remote from reality, in which all the axial vector currents are conserved as well as the vector ones.

\section{BROKEN SYMMETRY-MESON SUPERMULTIPLETS}

We know that because of isotopic spin conservation the baryons and mesons form degenerate isotopic multiplets, each corresponding to an irreducible representation of the isotopic spin algebra (3.1). Each multiplet has $2 I+1$ components, where the quantum num-

\footnotetext{
${ }^{32}$ M. Gell-Mann, California Institute of Technology Synchro-
} tron Laboratory Report No. CTSL-20, 1961 (unpublished).

${ }^{33}$ Y. Ne'eman, Nuclear Phys. 26, 222 (1961).

${ }^{34}$ A. Salam and J. C. Ward, Nuovo cimento 20, 419 (1961). ber $I$ distinguishes one representation from another and gives us the eigenvalue $I(I+1)$ of the operator $\sum_{i=1}^{3} I_{i}^{2}$, which commutes with all the elements of the isotopic spin group. The operators $I_{i}$ are represented, within the multiplet, by Hermitian $(2 I+1) \times(2 I+1)$ matrices having the commutation rules (3.1) of the algebra.

If we start from the doublet representation, we can build up all the others by considering combinations of particles that transform like the original doublet. Just as $(p, n)$ form a doublet representation for which the $I_{i}$ are represented by $\tau_{i} / 2$, the antiparticles $(\bar{n},-\bar{p})$ also form a doublet representation that is equivalent. (Notice the minus sign on the antiproton state or field.) Now, if we put together a nucleon and an antinucleon, we can form the combination

$$
\bar{N} N=\bar{p} p+\bar{n} n,
$$

which transforms like an isotopic singlet, or the combinations

$$
\bar{N} \tau_{i} N, \quad(i=1,2,3)
$$

which form an isotopic triplet. The direct product of nucleon and antinucleon doublets gives us a singlet and a triplet. Any meson that can dissociate virtually into nucleon and antinucleon must be either a singlet or a triplet. For the singlet state, the components $I_{i}$ are all zero, while for the three triplet states the three $3 \times 3$ matrices, $I_{i}{ }^{j k}$ of the components $I_{i}$, are given by

$$
I_{i}{ }^{i k}=-i e_{i j k} \text {. }
$$

Now let us generalize these familiar results to the unitary spin and the three basic baryons $b$ (comprising $n, p$, and $\Lambda$ ). These three fields or particles form a threedimensional irreducible representation of the unitary spin algebra (4.16) from which all the other representations may be constructed.

For example, consider a meson that can dissociate into $b$ and $\bar{b}$. It must transform either like

$$
\bar{b} b=\bar{p} p+\bar{n} n+\bar{\Lambda} \Lambda,
$$

a unitary singlet, or else like

a unitary octet.

$$
\bar{b} \lambda_{i} b, \quad(i=1, \cdots, 8)
$$

The unitary singlet is evidently neutral, with strangeness $S=0$, and forms an isotopic singlet. But how does the unitary octet behave with respect to isotopic spin? We form the combinations

$$
\begin{aligned}
& \left.\begin{array}{rl}
\bar{b}\left(\lambda_{1}-i \lambda_{2}\right) b / 2 & =\bar{n} p, \\
\bar{b} \lambda_{3} b / \sqrt{2} & =(\bar{p} p-\bar{n} n) / \sqrt{2}, \\
\bar{b}\left(\lambda_{1}+i \lambda_{2}\right) b / 2 & =\bar{p} n,
\end{array}\right\} \quad I=1, S=0 \\
& \left.\left.\begin{array}{l}
\bar{b}\left(\lambda_{4}-i \lambda_{5}\right) b / 2=\bar{\Lambda} p, \\
\bar{b}\left(\lambda_{6}-i \lambda_{7}\right) b / 2=\bar{\Lambda} n,
\end{array}\right\} \quad\right\} \quad=\frac{1}{2}, S=+1 \\
& \left.\left.\begin{array}{l}
\bar{b}\left(\lambda_{4}+i \lambda_{5}\right) b / 2=\bar{p} \Lambda, \\
\bar{b}\left(\lambda_{6}+i \lambda_{7}\right) b / 2=\bar{n} \Lambda,
\end{array}\right\} \quad\right\}=\frac{1}{2}, S=-1 \\
& \bar{b} \lambda_{8} b / \sqrt{2}=(\bar{p} p+\bar{n} n-2 \bar{\Lambda} \Lambda) / \sqrt{ } 6, \quad I=0, S=0,
\end{aligned}
$$


and we see immediately that the unitary octet comprises an isotopic triplet with $S=0$, a pair of isotopic doublets with $S= \pm 1$, and an isotopic singlet with $S=0$. All these are degenerate only in the limit of unitary symmetry $\left(L^{\prime \prime}=0\right)$; when the mass-splitting term is turned on, the singlet, the triplet, and the pair of doublets should have three somewhat different masses.

The known pseudoscalar mesons $(\pi, K$, and $\bar{K})$ fit very well into this picture, provided there is an eighth pseudoscalar meson to fill out the octet. Let us call the hypothetical isotopic singlet pseudoscalar meson $\chi^{0}$. Since it is pseudoscalar, it cannot dissociate (virtually or really) into $2 \pi$. It has the value +1 for the quantum number $G$, so that it cannot dissociate into an odd number of pions either. Thus in order to decay by means of the strong interactions, it must have enough energy to yield $4 \pi$. It would then appear as a $4 \pi$ resonance. The decay into $4 \pi$ is, however, severely hampered by centrifugal barriers.

If the mass of $\chi^{0}$ is too low to permit it to decay readily into $4 \pi$, then it will decay electromagnetically. If there is sufficient energy, the decay mode $\chi^{0} \rightarrow \pi^{+}+\pi^{-}+\gamma$ is most favorable; otherwise $e^{34 a}$ it will decay into $2 \gamma$ like $\pi^{0}$.

Let us now turn to the vector mesons. The best known vector meson is the $I=1, J=1^{-}$resonance of $2 \pi$, which we shall call $\rho$. It has a mass of about 750 Mev. According to our scheme, it should belong, like the pion, to a unitary octet. Since it occupies the same position as the $\pi(I=1, S=0)$, we denote it by the succeeding letter of the Greek alphabet.

The vector analog of $\chi^{0}$ we shall call $\omega^{0}$ (skipping the Greek letter $\psi$ ). It must have $I=0, J=1^{-}$, and $G=-1$ and so it is capable of dissociation into $\pi^{+}+\pi^{-}+\pi^{0}$. Presumably it is the $3 \pi$ resonance found experimentally ${ }^{35}$ at about $790 \mathrm{Mev}$.

In order to complete the octet, we need a pair of strange doublets analogous to $K$ and $\bar{K}$. In the vector case, we shall call them $M$ and $\bar{M}$ (skipping the letter $L)$. Now there is a known $K \pi$ resonance with $I=\frac{1}{2}$ at about $884 \mathrm{Mev}$. If it is a $p$-wave resonance, then it fits the description of $M$ perfectly.

In the limit of unitary symmetry, we can have, besides the unitary octet of vector mesons, a unitary singlet. The hypothetical $B^{0}$ that we discussed in Sec. III would have such a character. If $B^{0}$ exists, then the turning-on of the mass-splitting term $L^{\prime \prime}$ mixes the states $B^{0}$ and $\omega^{0}$, which are both isotopic singlets.

Other mesons may exist besides those discussed, for example, scalar and axial vector mesons. All those that can associate into $b+\bar{b}$ should form unitary octets or

${ }^{34 a}$ Note added in proof. H. P. Duerr and W. Heisenberg (preprint) have pointed out the importance of the decay mode $\chi^{0} \rightarrow 3 \pi$ induced by electromagnetism. For certain $\chi$ masses, it may be a prominent mode.

${ }^{35}$ B. C. Maglić, L. W. Alvarez, A. H. Rosenfeld, and M. L. Stevenson, Phys. Rev. Letters 7, 178 (1961).
TABLE III. Possible meson octets and singlets.

\begin{tabular}{ccccccc}
\hline \hline $\begin{array}{c}\text { Unitary } \\
\text { spin }\end{array}$ & $\begin{array}{c}\text { Isotopic } \\
\text { spin }\end{array}$ & $\begin{array}{c}\text { Strange- } \\
\text { ness }\end{array}$ & $\begin{array}{c}\text { Pseudo- } \\
\text { scalar }\end{array}$ & Vector & Scalar & $\begin{array}{c}\text { Axial } \\
\text { vector }\end{array}$ \\
\hline Octet & 1 & 0 & $\pi$ & $\rho$ & $\pi^{\prime}$ & $\rho^{\prime}$ \\
& $1 / 2$ & +1 & $K$ & $M$ & $K^{\prime}$ & $M^{\prime}$ \\
& $1 / 2$ & -1 & $\bar{K}$ & $\bar{M}$ & $\bar{K}^{\prime}$ & $\bar{M}^{\prime}$ \\
& 0 & 0 & $\chi$ & $\omega$ & $\chi^{\prime}$ & $\omega^{\prime}$ \\
Singlet & 0 & 0 & $A$ & $B$ & $A^{\prime}$ & $B^{\prime}$ \\
\hline
\end{tabular}

singlets or both, with each octet splitting into isotopic multiplets because of the symmetry-breaking term $L^{\prime \prime}$.

A list of some possible meson states is given in Table III, along with suggested names for the mesons.

It is interesting that we can predict not only the degeneracy of an octet in the $\operatorname{limit} L^{\prime \prime} \rightarrow 0$ but also a sum rule $^{32}$ that holds in first order in $L^{\prime \prime}$ :

$$
\begin{aligned}
\left(m_{K}+m_{\bar{K}}\right) / 2 & =\left(3 m_{\chi}+m_{\pi}\right) / 4, \\
\left(m_{M}+m_{\bar{M}}\right) / 2 & =\left(3 m_{\omega}+m_{\rho}\right) / 4 .
\end{aligned}
$$

If $M$ is at about $884 \mathrm{Mev}$ and $\rho$ at about $750 \mathrm{Mev}$, then $\omega$ should lie at about $930 \mathrm{Mev}$ according to the sum rule; since it is actually at $790 \mathrm{Mev}$, the sum rule does not seem to give a good description of the splitting. Perhaps an important effect is the repulsion between the $\omega^{0}$ and $B^{0}$ levels, pushing $\omega^{0}$ down and $B^{0}$ up. For what it is worth, (6.3) gives a $\chi^{0}$ mass of around 610 Mev.

In the limit of unitary symmetry, not only are the supermultiplets degenerate but their effective couplings are symmetrical. For example, the effective coupling of the unitary pseudoscalar octet to $N$ and $\Lambda$ takes the form

$$
\begin{aligned}
i g_{1}\left\{\bar{N} \tau \gamma_{5} N \cdot \pi+\bar{N}\right. & \gamma_{5} \Lambda K+\bar{\Lambda} \gamma_{5} N \bar{K} \\
& \left.+3^{-\frac{1}{2}} \bar{N} \gamma_{5} N \chi-2 \times 3^{-\frac{1}{2}} \bar{\Lambda} \gamma_{5} \Lambda \chi\right\}
\end{aligned}
$$

in terms of renormalized "fields." Now, as the term $L$ " is turned on, the various coupling constants become unequal; instead of calling them all $g_{1}$, we refer to them as $g_{N N \pi}, g_{N \Lambda K}, g_{N N \chi}$, and $g_{\Lambda \Lambda \chi}$, respectively, each of these constants being the measurable renormalized coupling parameter at the relevant pole.

We have written the effective coupling (6.4) as if there were renormalized fields for all the particles involved, but that is only a matter of notation; the mesons can perfectly well be composite. We may simplify the notation still further by constructing a traceless $3 \times 3$ matrix II containing the pseudoscalar "fields" in such a way that (6.4) becomes

$$
i g_{1} \bar{b} \Pi \gamma_{5} b
$$

We may now write, in a trivial way, other effective couplings in the limit of unitary symmetry. We define a traceless $3 \times 3$ matrix $W_{\alpha}$ containing the "fields" for the vector meson octet just as $\Pi^{*}$ contains those for the pseudoscalar octet. We then have the invariant 
effective coupling

$$
i \gamma_{1} \operatorname{Tr} W_{\alpha}\left(\Pi \partial_{\alpha} \Pi-\partial_{\alpha} \Pi \Pi\right) / 2
$$

in the symmetric limit. When the asymmetry is turned on, the single coupling parameter $\gamma_{1}$ is replaced by the set of different parameters $\gamma_{\rho \pi \pi}, \gamma_{\rho K K}, \gamma_{\omega K K}, \gamma_{M K \pi}$, and $\gamma_{M K_{X}}$.

In the same way, we have another effective coupling

$$
i h_{1} \operatorname{Tr} \Pi\left(\partial_{\alpha} W_{\beta}-\partial_{\beta} W_{\alpha}\right)\left(\partial_{\gamma} W_{\delta}-\partial_{\delta} W_{\gamma}\right) e_{\alpha \beta \gamma \delta}
$$

in the symmetric limit; in the actual asymmetric case, we define the distinct constants $h_{\pi \omega \rho}, h_{\pi M M}, h_{\chi \omega \omega}, h_{\chi \rho \rho}$, $h_{\chi M M}, h_{K M \rho}$, and $h_{K M \omega}$. All of these constants can be measured, in principle, in "pole" experiments, except that for the broad resonances like $\rho$ the poles are well off the physical sheet.

We have generalized the definitions of constants like $g_{N N \pi}$ and $\gamma_{\rho \pi \pi}$, as used in Sec. II, to other particles. The constants $\gamma_{\rho}$ and $f_{\pi}$ of Sec. II also have analogs, of course, and we define $\gamma_{\omega}, f_{K}$, etc., in the obvious way. In the limit of unitary symmetry, of course, we would have $f_{\pi}=f_{K}=f_{\chi}$ and $\gamma_{\rho}=\gamma_{\omega}=\gamma_{M}$. Likewise, the constant $-G_{A} / G$ for nucleon $\beta$ decay would equal the corresponding quantity $-G_{A}{ }^{\Lambda N} / G$ for the $\beta$ decay of $\Lambda$.

\section{BROKEN SYMMETRY-BARYON SUPERMULTIPLETS}

What has been done in the previous section may be described mathematically as follows. We considered a three-dimensional representation of the unitary spin algebra (4.16) or of the group $S U(3)$ that is generated by the algebra. It is the representation to which $b$ belongs (that is, $n, p$, and $\Lambda$ ) and we may denote it by the symbol 3 .

The antiparticles $\bar{b}$ belong to the conjugate representation $3^{*}$, which is inequivalent ${ }^{36}$ to 3 . We have then taken the direct product $3 \times 3^{*}$ and found it to be given by the rule

$$
3 \times 3^{*}=8+1,
$$

where $\mathbf{8}$ is the octet representation and $\mathbf{1}$ the singlet representation of unitary spin. Each of these is its own conjugate; that is a situation that occurs only when the dimension is the cube of an integer.

There are, of course, more complicated representations to which mesons might belong that are incapable (in the limit of unitary symmetry) of dissociation into $b+\bar{b}$ but capable of dissociation into $2 b+2 \bar{b}$ or higher configurations. But we might guess that at least the mesons of lowest mass would correspond to the lowest configurations.

Now we want to examine the simplest configurations

${ }^{36}$ In other words, no unitary transform can convert the representations 3 and $3^{*}$ into each other. That is easy to see, since the eigenvalues of $\lambda_{8}$ are opposite in sign for the two representations, and changing the signs changes the set of eigenvalues. In the case of the group $S U(2)$ of isotopic spin transformations, the basic spinor representation $I=\frac{1}{2}$ is equivalent to the corresponding antiparticle representation. for baryons, apart from just $b$. Evidently the next simplest is $2 b+\bar{b}$, which poses the problem of reducing the direct product $3 \times 3 \times 3^{*}$; the result is the following:

$$
3 \times 3 \times 3^{*}=3 \times 1+3 \times 8=3+3+6+15 .
$$

The six-dimensional representation $\mathbf{6}$ is composed of an isotopic triplet with $S=-1$, a doublet with $S=0$, and a singlet with $S=+1$; the fifteen-dimensional representation $\mathbf{1 5}$ is composed of a doublet with $S=-2$, a singlet and a triplet with $S=-1$, a doublet and a quartet with $S=0$, and a triplet with $S=+1$.

According to the scheme, then, $\Xi$ should belong to $\mathbf{1 5}$. Where are the other members of the supermultiplet? For $S=-1$ and $S=0$, there are many known resonances, some of which might easily have the same spin and parity as $\Xi$. For $S=+1, I=1$, however, no resonance has been found so far (in $K^{+}-p$ scattering, for example).

The hyperon $\Sigma$ should also be placed in a supermultiplet, which may or may not be the same one to which $\Xi$ belongs; we do not know if the spin and parity of $\Sigma$ and $\Xi$ are the same, with $K$ taken to be pseudoscalar. If $\Sigma$ belongs to 6 in the limit of unitary symmetry, then there should be a $K N$ resonance in the $I=0$ state.

It is difficult to say at the present time if the baryon states can be reconciled with the model. Further knowledge of the baryon resonances is required.

One curious possibility is that the fundamental objects $b$ are hidden and that the physical $N$ and $\Lambda$, instead of belonging to 3 , belong, along with $\Sigma$ and $\Xi$, to the representation $\mathbf{1 5}$ in the limit of unitary symmetry. That would require the spins and parities of $N, \Lambda, \Sigma$, and $\Xi$ to be equal, and it would require a $\pi N$ resonance in the $p_{\frac{1}{2}}, I=\frac{3}{2}$ state as well as a $K N$ resonance in the $p_{\frac{1}{2}}, I=1$ state to fill out the supermultiplet.

\section{THE "EIGHTFOLD WAY"}

Unitary symmetry may be applied to the baryons in a more appealing way if we abandon the connection with the symmetrical Sakata model and treat unitary symmetry in the abstract. (An abstract approach is, of course, required if there are no "elementary" baryons and mesons.) Of all the groups that could be generated by the vector weak currents, $S U(3)$ is still the smallest and the one that most naturally gives rise to the rules $|\Delta \mathbf{I}|=\frac{1}{2}$ and $\Delta S / \Delta Q=0,+1$.

There is no longer any reason for the baryons to belong to the 3 representation or the other spinor representations of the group $S U(3)$; the various irreducible spinor representations are those obtained by reducing direct products like $3 \times 3 \times 3^{*}, 3 \times 3 \times 3 \times 3^{*} \times 3^{*}$, etc.

Instead, the baryons may belong, like the mesons, to representations such as $\mathbf{8}$ or $\mathbf{1}$ obtained by reducing the direct products of equal numbers of 3's and $3^{*}$ 's. It is then natural to assign the stable and metastable baryons $N, \Lambda, \Sigma$, and $\Xi$ to an octet, degenerate in the limit of unitary symmetry. We thus obtain the scheme 
of Gell-Mann" and Ne'eman" ${ }^{32}$ that we call the "eightfold way." The component $F_{8}$ of the unitary spin is now $(\sqrt{3} / 2) Y$, where $Y$ is the hypercharge (equal to strangeness plus baryon number).

The baryons of the octet must have the same spin and parity (treating $K$ as pseudoscalar). To first order in the violation of unitary symmetry, the masses should obey the sum rule analogous to (6.3):

$$
\left(m_{N}+m_{\Xi}\right) / 2=\left(3 m_{\Lambda}+m_{\Sigma}\right) / 4,
$$

which agrees surprisingly well with observations, the two sides differing by less than $20 \mathrm{Mev}$.

To form mesons that transform like combinations of these baryons and their antiparticles, we reduce the direct product $8 \times 8$ (remembering that $8=8^{*}$ ) and obtain

$$
8 \times 8=1+8+8+10+10 *+27,
$$

where 1 and 8 are the singlet and octet representations already discussed; 10 consists of an isotopic triplet with $Y=0$, a doublet with $Y=-1$, a quartet with $Y=+1$, and a singlet with $Y=-2 ; 10^{*}$ has the opposite behavior with respect to $Y$; and 27 consists of an isotopic singlet, triplet, and quintet with $Y=0$, a pair of doublets with $Y= \pm 1$, a pair of quartets with $Y= \pm 1$, and a pair of triplets with $Y= \pm 2$. Evidently the known mesons are to be assigned to octets and perhaps singlets, as in Sec. VI. The meson-nucleon scattering resonances must then also be assigned representations among those in (8.2); the absence so far of any observed structure in $K-N$ scattering makes it difficult to place the $I=3 / 2$, $J=3 / 2, \pi-N$ resonance in a supermultiplet.

The fact that 8 occurs twice in Eq. (8.2) means that there are two possible forms of symmetrical Yukawa coupling of a meson octet to the baryon octet in the limit of unitary symmetry. As in Sec. VI for the mesons, we form a $3 \times 3$ traceless matrix out of the formal "fields" of the baryon octet; call it $\$$. The effective symmetrical coupling of pseudoscalar mesons may then be written as

$$
\begin{aligned}
& i g_{1} \alpha \operatorname{Tr}\left(\bar{ß} \pi \gamma_{5} \Theta+\pi \bar{\otimes} \gamma_{5}() / 2\right. \\
& +i g_{1}(1-\alpha) \operatorname{Tr}\left(\bar{B} \pi \gamma_{5}\left(B-\pi \bar{\triangle} \gamma_{5} B\right) / 2 .\right.
\end{aligned}
$$

The two types of coupling differ in their behavior under the operation $R$ that exchanges $N$ and $\Xi, K$ and $\bar{K}$, $M$ and $\bar{M}$, etc.; the first term is symmetric while the second is antisymmetric under $R$. The parameter $\alpha$ just specifies how much of each effective coupling is presented in the limit of unitary symmetry. When we take into account violations of the symmetry, we must define separate coupling constants $g_{N N \pi}, g_{N K \Lambda}$, etc., in a suitable way.

Likewise the vector mesons have the general symmetrical coupling

$$
\begin{aligned}
& i \gamma_{1} \beta \operatorname{Tr}\left(\bar{B} W_{\alpha} \gamma_{\alpha} B+W_{\alpha} \bar{B} \gamma_{\alpha}(B)\right. \\
& +i \gamma_{1}(1-\beta) \operatorname{Tr}\left(\bar{B} W_{\alpha} \gamma_{\alpha} \beta-W_{\alpha} \bar{\Theta} \gamma_{\alpha} B\right),
\end{aligned}
$$

where we ignore Pauli moment terms for simplicity. To the extent that the vector meson octet $W_{\alpha}$ dominates the dispersion relations for the unitary spin current $\mathfrak{F}_{i \alpha}$, then the mesons of $W_{\alpha}$ are coupled effectively to the components of $\mathcal{F}_{i \alpha}$, and we have $\beta=0$ in (8.5). Then $\rho$ is effectively coupled to the isotopic spin current, $\omega$ to the hypercharge current, and $M$ to the strangenesschanging vector current. 'The first two of these currents are conserved, and so we have the approximate universality of $\rho$ and $\omega$ couplings proposed by Sakurai ${ }^{11}$ and discussed in Sec. II. In the limit of unitary symmetry, under the assumptions just mentioned, $\rho$ is effectively coupled to the current of $2 \gamma_{1} \mathbf{I}$ and $\omega$ to the current of $2 \gamma_{1} F_{8}=\sqrt{3} \gamma_{1} Y$.

The electromagnetic current is now given by the formula

$$
j_{\alpha}=\mathfrak{F}_{3 \alpha}+3^{-\frac{1}{2}} \mathfrak{F}_{8 \alpha}
$$

instead of (5.4), while the weak vector current is still described by Eq. (5.5). If we are to treat the vector and axial vector currents by means of $S U(3) \times S U(3)$, as we did earlier, then the entire weak current is given by (5.22) and we have the commutation rules (5.3), (5.9), and (5.10) for the various components of the currents. The question of the behavior of $H$ under the group $S U(3) \times S E$ (3) should, however, be re-examined for the cightfold way; we shall not go into that question here. But let us consider how the baryon octet transforms in the limit of conserved vector and axial vector currents [invariance under $S U(3) \times S U(3)]$. In the Sakata model, the left-handed baryons transformed under $\left(F_{i}{ }^{+}, F_{j}^{-}\right)$ like $(3,1)$, while the right-handed baryons transformed according to $(\mathbf{1}, \mathbf{3})$. For the eightfold way, there are two simple possibilities for these transformation properties. Either we have $(8,1)$ and $(1,8)$ or else we adjoin a ninth neutral baryon (which need not be degenerate with the other eight in the limit of conserved vector currents and which need not have the same parity) and use the transformation properties $\left(\mathbf{3}, \mathbf{3}^{*}\right)$ and $\left(3^{*}, 3\right)$. In the first case, the baryons transform like the quantities $\mathfrak{F}_{i a}$ and $\mathfrak{F}_{i \alpha}{ }^{5}(i=1, \cdots, 8)$ and in the second case they transform like $u_{i}$ and $v_{i}(i=0, \cdots, 8)$ of Sec. V.

\section{REMARKS AND SUGGESTIONS}

Our approach to the problem of baryon and meson couplings leads to a number of suggestions for new investigations, both theoretical and experimental.

First, the equal-time commutation relations for currents and densities lead to exact sum rules for the weak and electromagnetic matrix elements. As an example, take the commutation rules (3.5) for the isotopic spin current. These do not, of course, depend on any higher symmetry, but they can be used to illustrate the results that can be obtained from the more general relations like (5.3).

Consider the electromagnetic form factor $F_{\pi}(s)$ of the charged pion, which is just the form factor of the 
isotopic spin current between one-pion states. Let $p$ and $p^{\prime}$ be the initial and final pion four-momenta, with $s=-\left(p-p^{\prime}\right)^{2}$. Let $K$ be any four-momentum with $K^{2}=-m_{\pi}{ }^{2}$. Then, taking the matrix element of (3.5) between one-pion states, we obtain the result

$$
\begin{aligned}
& 2\left(p_{0}+p_{0}{ }^{\prime}\right) K_{0} F_{\pi}\left(-\left(p-p^{\prime}\right)^{2}\right) \\
& \quad=\left(p_{0}+K_{0}\right)\left(p_{0}{ }^{\prime}+K_{0}\right) F_{\pi}\left(-(p-K)^{2}\right) F_{\pi}\left(-\left(p^{\prime}-K\right)^{2}\right) \\
& \quad-\left(p_{0}-K_{0}\right)\left(p_{0}{ }^{\prime}-K_{0}\right) F_{\pi}\left(-(p+K)^{2}\right) F_{\pi}\left(-\left(p^{\prime}+K\right)^{2}\right)
\end{aligned}
$$$$
\text { +inelastic terms, }
$$

where the inelastic terms come from summing over bilinear forms in the inelastic matrix elements of the current. We see that if there is no inelasticity the form factor is unity. Thus the departure from unity of $F_{\pi}(s)$ is related to the amount of inelasticity.

A similar relation is familiar in nonrelativistic quantum mechanics:

$$
\left\langle e^{i\left(\mathrm{p}-\mathrm{p}^{\prime}\right) \cdot \mathbf{x}}\right\rangle_{00}=\sum_{n}\left\langle e^{i(\mathrm{p}-\mathrm{k}) \cdot \mathbf{x}}\right\rangle_{0 n}\left\langle e^{i\left(\mathrm{k}-\mathrm{p}^{\prime}\right) \cdot \mathbf{x}}\right\rangle_{n 0} .
$$

If we apply relations like (9.1) to the matrix elements of non-conserved currents like $\mathbf{P}_{\boldsymbol{\alpha}}$, along with the linear homogeneous dispersion relations for these matrix elements, we can in principle determine constants like $-G_{A} / G$.

A second line of theoretical investigation is suggested by the vanishing at high momentum transfer of matrix elements of divergences of non-conserved currents, like $\partial_{\alpha} \mathbf{P}_{\alpha}$. We should try to find limits involving high energies and high momentum transfers in which we can show that the conservation of helicity, unitary spin, etc., becomes valid. A preliminary effort in this direction has been made by Gell-Mann and Zachariasen. ${ }^{37}$

A third topic of study is the testing of broken symmetry at low energy. Do the mesons fall into unitary octets and singlets? An experimental search for $\chi^{0}$ is required and also a determination of the spin of $K^{*}$ at $884 \mathrm{Mev}$ to see if it really is our $M$ meson.

Let us discuss briefly the properties of $\chi^{0}$. An $I=0$ state of $4 \pi$ can have two types of symmetry: either totally symmetric (partition [4]) in both space and isotopic spin or else the symmetry of the partition $[2+2]$ in space and in isotopic spin. For a pseudoscalar state, the first type of wave function in momentum space is very complicated. If $\mathbf{p}, \mathbf{q}$, and $\mathbf{r}$ are the three momentum differences, it must look like

$$
\begin{aligned}
\mathbf{p} \cdot \mathbf{q} \times \mathbf{r}\left(E_{1}-E_{2}\right)\left(E_{2}-E_{3}\right) & \left(E_{3}-E_{4}\right) \\
& \times\left(E_{1}-E_{3}\right)\left(E_{1}-E_{4}\right)\left(E_{2}-E_{4}\right),
\end{aligned}
$$

times a symmetric function of the energies $E_{1}, E_{2}, E_{3}, E_{4}$ of the four pions. On the basis of any reasonable dynamical picture of $\chi^{0}$, such a wave function should have a very small amplitude. In particular, dispersion theory suggests that the wave function of $\chi^{0}$ should have large contributions from virtual dissociation into $2 \rho$, which gives a wave function with $[2+2]$ symmetry.

${ }^{37}$ M. Gell-Mann and F. Zachariasen, Phys. Rev. 123, 1065 (1961).
If $[2+2]$ predominates, then the charge ratio in decay is $2: 1$ in favor of $2 \pi^{0}+\pi^{+}+\pi^{-}$over $2 \pi^{+}+2 \pi^{-}$, with $4 \pi^{0}$ absent. If virtual dissociation into $2 \rho$ actually predominates, then the matrix element of the $4 \pi$ configuration is easily written down and the spectrum of the decay $\chi^{0} \rightarrow 4 \pi$ can be calculated.

If $\chi^{0}$ is lighter than $4 \pi$, it will, of course, decay electromagnetically. Even if it is above threshold for $4 \pi$, however, the matrix element for decay contains so many powers of pion momenta that electromagnetic decay should be appreciable over a large range of masses. The branching ratio $\left(\pi^{+}+\pi^{-}+\gamma\right) /(4 \pi)$ is approximately calculable by dispersion methods. In both cases $\chi^{0}$ first dissociates into $2 \rho$. Then either both virtual $\rho$ mesons decay into $2 \pi$, or else (in the case where both are neutral) one may decay into $\pi^{+}+\pi^{-}$, while the other turns directly into $\gamma$. If we draw a diagram for such a process, then the constant $\gamma_{\rho \pi \pi}$ is inserted whenever we have a $\rho \pi \pi$ vertex and the constant $e m_{\rho}^{2} / 2 \gamma_{\rho}$ at a $\rho-\gamma$ junction. ${ }^{10}$

If the meson spectrum is consistent with broken unitary symmetry, we should examine the baryons, and see whether the various baryon states fit into the represensations 3, 6, and 15 (or the representations $\mathbf{1}, \mathbf{8}$, $10,10 *$, and 27 that arise in the alternative form of unitary symmetry).

If some states are lacking in a given supermultiplet, it does not necessarily prove that the broken symmetry is wrong, but only that it is badly violated. We assume that baryon isobars like the $\pi N \frac{3}{2}, \frac{3}{2}$ resonance are dynamical in nature; there may be some attractive and some repulsive forces in this channel, and the attractive ones have won out, producing the resonance. In the $K N$ channel with $I=1$, for example, it is conceivable that the repulsive ones are stronger (because of symmetry violation), and the analogous $p_{\frac{3}{2}}$ resonance disappears. In such a situation, the concept of broken symmetry at low energies is evidently of little value.

Suppose, however, that the idea of broken unitary symmetry is confirmed for both mesons and baryons, say according to the Sakata picture, in which $N$ and $\Lambda$ belong to the representation 3 in the limit of unitary symmetry. There are, nevertheless, gross violations of unitary symmetry, and the elucidation of these, both theoretical and experimental, is a fourth interesting subject.

If unitary symmetry were exact, then not only would $m_{K} / m_{\pi}$ equal unity, instead of about 3.5 , but $f_{K}{ }^{2} / f_{\pi}^{\prime}{ }^{2}$ would be 1 instead of about 6 , and $3 G_{A}{ }^{2}+G_{V}{ }^{2}$ for the $\beta$ decay of $\Lambda$ would be equal to $3 G_{A}{ }^{2}+G_{V}{ }^{2}$ for the nucleon instead of being $1 / 15$ as large. All these huge departures from unity represent very serious violations of unitary symmetry.

Y'et the relatively small mass difference of $N$ and $\Lambda$ compared to their masses would seem to indicate, if our model is right, that the constant $c$ in Eq. (5.21) is considerably smaller than unity. It is conceivable that the large mass ratio of $K$ to $\pi$ comes about because the total 
mass of the system is so small. It is possible that even with a fairly small $c$ (say $\sim-\frac{1}{10}$ ) we might explain the gross violations of unitary symmetry. We might try to interpret the large values of $g_{N N \pi}{ }^{2} / g_{N K \Lambda}{ }^{2}, f_{K}{ }^{2} / f_{\pi}^{2}$, etc., in terms of the large value of $m_{K}^{2} / m_{\pi}^{2}$.

An example of such a calculation, and one that illustrates the various methods suggested in this article, is the following. We try to calculate $f_{K}{ }^{2} / f_{\pi}^{2}$ in terms of $m_{K}{ }^{2} / m_{\pi}^{2}$.

Consider the following vacuum expectation value, written in parametric representation:

$$
\begin{array}{r}
\left\langle\left[\mathcal{F}_{1 \alpha}{ }^{5}(x), \partial_{\beta} \mathcal{F}_{1 \beta}{ }^{5}\left(x^{\prime}\right)\right]\right\rangle_{0}=i /(2 \pi)^{3} \int d^{4} K e^{i K \cdot\left(x-x^{\prime}\right)} \\
\times K_{\alpha} \epsilon(K) \int d M^{2} / M^{2} \delta\left(K^{2}+M^{2}\right) \rho\left(M^{2}\right)
\end{array}
$$

Here $x$ and $x^{\prime}$ are arbitrary space-time points. In terms of (9.3), we have

$$
\begin{array}{r}
\left\langle\left[\partial_{\alpha} F_{1 \alpha}{ }^{5}(x), \partial_{\beta} F_{1 \beta}{ }^{5}\left(x^{\prime}\right)\right]\right\rangle=1 /(2 \pi)^{3} \int d^{4} K e^{i K \cdot\left(x-x^{\prime}\right)} \\
\times \epsilon(K) \int d M^{2} \delta\left(K^{2}+M^{2}\right) \rho\left(M^{2}\right)
\end{array}
$$

Now the contribution of the one-pion intermediate state is easily obtained in terms of the constant $f_{\pi}^{2}$ :

$$
\rho\left(M^{2}\right)=\delta\left(M^{2}-m_{\pi}^{2}\right) m_{\pi}^{4} / 4 f_{\pi}^{2}+\text { higher terms. }
$$

If $\int \rho\left(M^{2}\right) d M^{2} / M^{2}$ converges and if the one-pion term dominates, we have

$$
\int \rho\left(M^{2}\right) d M^{2} / M^{2} \approx m_{\pi}^{2} / 4 f_{\pi}^{2} .
$$

But from (9.3) we can extract the expectation value of the equal-time commutator of the fourth component of $\mathfrak{F}_{1 \alpha}{ }^{5}$ with $\partial_{\beta} \mathfrak{F}_{1 \beta}{ }^{5}$; making use of (5.20) and (5.24), we can express the result in terms of $\left\langle u_{0}\right\rangle$ and $\left\langle u_{8}\right\rangle$. Thus we find

$$
\begin{aligned}
\int \rho\left(M^{2}\right) d M^{2} / M^{2}= & {\left[(2 / 3)^{\frac{1}{2}}+(1 / 3)^{\frac{1}{2}} c\right] } \\
& \times\left[(2 / 3)^{\frac{1}{2}}\left\langle u_{0}\right\rangle_{0}+(1 / 3)^{\frac{1}{2}}\left\langle u_{8}\right\rangle_{0}\right],
\end{aligned}
$$

assuming convergence.

Now we can do exactly the same thing for $\mathfrak{F}_{4 \alpha}{ }^{5}$ and the $K$ meson, obtaining, in place of the formula

$$
m_{\pi}^{2} / 4 f_{\pi}^{2} \approx\left[\left(\frac{2}{3}\right)^{\frac{1}{2}}+\left(\frac{1}{3}\right)^{\frac{1}{2}} c\right]\left[\left(\frac{2}{3}\right)^{\frac{1}{2}}\left\langle u_{0}\right\rangle_{0}+\left(\frac{1}{3}\right)^{\frac{1}{2}}\left\langle u_{8}\right\rangle_{0}\right],
$$

the analogous result

$$
m_{K}{ }^{2} / 4 f_{K}{ }^{2} \approx\left[\left(\frac{2}{3}\right)^{\frac{1}{2}}-\left(\frac{1}{12}\right)^{\frac{1}{2}} c\right]\left[\left(\frac{2}{3}\right)^{\frac{1}{2}}\left\langle u_{0}\right\rangle_{0}-\left(\frac{1}{12}\right)^{\frac{1}{2}}\left\langle u_{8}\right\rangle_{0}\right] \text {. }
$$

If $c$ is really small, presumably $\left\langle u_{8}\right\rangle_{0}$ is also small compared to $\left\langle u_{0}\right\rangle_{0}$. Then we can, roughly, set (9.8) equal to (9.9), obtaining

$$
f_{K}^{2} / f_{\pi}^{2} \approx m_{K}^{2} / m_{\pi}^{2}
$$

The left-hand side is about 6 and the right-hand side about 10 . Thus we can, in a crude approximation, calculate the rate of $K^{+} \rightarrow \mu^{+}+\nu$ in terms of that for $\pi^{+} \rightarrow$ $\mu^{+}+\nu$ and explain one large violation of symmetry in terms of another.

The Goldberger-Treiman formula relating $f_{\pi}, g_{N N \pi}$, and $\left(-G_{A} / G\right)$ can also be used for the $K$ particle to give a relation among $f_{K}, g_{N \Lambda K}$, and $\left(-G_{A} / G\right)$ for the $\beta$ decay of $\Lambda$. Of course, the $K$-particle pole is much closer to the branch line beginning at $\left(m_{K}+2 m_{\pi}\right)^{2}$ than the pion pole is to the branch line beginning at $9 m_{\pi}^{2}$; thus the Goldberger-Treiman formula may be quite bad for the $K$ meson. Still, we may try to use it to discuss the coupling of $N$ and $\Lambda$ to $K$ and to leptons. We have

$$
\left(m_{N}+m_{\Lambda}\right)\left(-G_{A}{ }^{N \Delta} / G\right) \approx g_{N \Lambda K} / f_{K},
$$

by analogy with (2.8). Comparing the two formulas, we have

$$
\begin{aligned}
& \left(-G_{A}{ }^{N \Lambda} / G\right)^{2}\left(-G_{A} / G\right)^{-2} \\
& \quad \approx g_{N \Lambda K^{2}} g_{N N \pi}{ }^{-2}\left(2 m_{N} f_{\pi}\right)^{2}\left[\left(m_{\Lambda}+m_{N}\right) f_{K}\right]^{-2} .
\end{aligned}
$$

The ratio of $g^{2}$ factors is thought to be $\sim 0.1$ from photoproduction of $K$, while the remaining factor on the right is also $\sim 0.1$, so that the Goldberger-Treiman relation leads us to expect a very small axial vector $\beta$-decay rate for the $\Lambda$, much smaller than the observed one. The observed $\beta$ decay would be nearly all vector; this prediction of the Goldberger-Treiman formula can easily be checked by observing the electron-neutrino angular correlation in the $\beta$ decay of $\Lambda$, using bubble chambers.

We should, of course, try to predict the value of

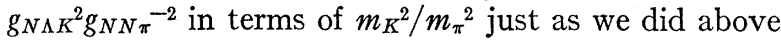
for $f_{K^{2}} / f_{\pi}^{2}$; however, it is a much harder problem.

When we know more about the coupling constants of the vector mesons (strong coupling constants such as $\gamma_{\omega N N}, h_{\omega \pi \rho}$, etc., and coupling strengths of currents such as $\gamma_{\omega}, \gamma_{M}$, etc.) we will be able to make a survey of the pattern of coupling constants as well as the pattern of masses and see whether the higher symmetry has any relevance. Also it should become clear how well the approximation of dominant low-mass states works, in terms of universality of meson couplings and Goldberger-Treiman relations. ${ }^{38}$

In summary, then, we suggest the use of the equaltime commutators to predict sum rules, attempts to derive high-energy conservation laws and to check them

${ }^{38}$ An interesting relation of the Goldberger-Treiman type is one that holds if the trace $\theta_{\alpha \alpha}$ of the stress-energy-momentum tensor has matrix elements obeying highly convergent dispersion relations. Because of the vanishing of the self-stress, the expectation value of $\theta_{\alpha \alpha}$ in the state of a particle at rest gives the mass of the particle. Rewriting the matrix element as one between the vacuum and a one-pair state, we see that the dispersion relation involves intermediate states with $I=0, J=0^{+}, G=+1$. If there is a resonance or quasi-resonance in this channel (like the $\chi^{\prime}$ meson of Table III) and if that resonance dominates the dispersion relation at low momentum transfers, then the coupling of the resonant state to different particles is roughly proportional to their masses. That is just the situation discussed by Schwinger in reference 1 and by Gell-Mann and Lévy in reference 17 for the " $\sigma$ meson." 
experimentally, the search for broken symmetry at low energies, attempts to calculate some violations in terms of others, and efforts to check the highly convergent dispersion relations dominated by low-mass states.

Nowhere does our work conflict with the program of Chew et al. of dynamical calculation of the $S$ matrix for strong interactions, using dispersion relations. If something like the Sakata model is correct, then most of the mesons are dynamical bound states or resonances, and their properties are calculable according to the program. Those particles for which there are fundamental fields (like $n, p, \Lambda$, and $B^{0}$ in the specific field-theoretic model) would presumably occur as CDD poles or resonances in the dispersion relations. ${ }^{39}$

If there are no fundamental fields and no CDD poles, all baryons and mesons being bound or resonant states of one another, models like that of Sakata will fail; the symmetry properties that we have abstracted can still be correct, however. This situation would presumably differ in two ways ${ }^{10}$ from the one mentioned above. First, all the masses and coupling constants could be calculated from coupled dispersion relations. Second, certain scattering amplitudes at high energies would show different behavior, corresponding to different kinds of subtractions in the dispersion relations. The second point should be investigated further, as it could lead to experimental tests of the "fundamental" character of various particles..$^{10,40}$

\section{ACKNOWLEDGMENTS}

It is a pleasure to thank R. P. Feynman, S. L. Glashow, and R. Block for many stimulating discussions of symmetry, and to acknowledge the great value of conversations with G. F. Chew, S. Frautschi, R. Haag, R. Schroer, and F. Zachariasen about the explanation of approximate universality in terms of highly convergent dispersion relations.

\section{APPENDIX}

The field theories of the Fermi-Yang and Sakata models, given by Eqs. (3.17) and (4.1), respectively, belong to a general class of theories, which we now describe.

The Lagrangian density $L$ is given as a function of a number of fields $\psi_{A}$ and their gradients. The kinetic part of the Lagrangian (consisting of those terms containing gradients) is invariant under a set of infinitesimal unitary transformations generated by $N$ independent Hermitian operators $R_{i}$, which may depend on the time. Under the transformations, the various fields $\psi_{A}$ undergo linear recombinations:

$$
\begin{array}{r}
\psi_{A}(\mathbf{x}, t) \rightarrow \psi_{A}(\mathbf{x}, t)-i \Lambda_{i}\left[R_{i}(t), \psi_{A}(\mathbf{x}, t)\right] \\
=\psi_{A}(\mathbf{x}, t)+i \Lambda_{i} \sum_{B} M_{i}^{A B} \psi_{B}(\mathbf{x}, t),
\end{array}
$$

${ }^{39}$ L. Castillejo, R. H. Dalitz, and F. J. Dyson, Phys. Rev. 101, 453 (1956)

${ }^{40}$ S. C. Frantschi, M. Gell-Mann, and F. Zachariasen (to be published). where $\Lambda_{i}$ is the infinitesimal gauge constant associated with the $i$ th transformation. The equal-time commutation rules of the $R_{i}$ are the same as those of the matrices $M_{i}$. Moreover, the set of $R_{i}$ and linear combinations of $R_{i}$ is algebraically complete under commutation; in other words, we have an algebra. The matrices $M_{i}$ are the basis of a representation of the algebra (in general, a reducible representation). It is convenient to take the matrices of the basis to be orthonormal,

$$
\operatorname{Tr} M_{i} M_{j}=(\text { const }) \delta_{i j}
$$

redefining the $R_{i}$ accordingly. The structure constants $c_{i j k}$ in the commutation rules

$$
\begin{aligned}
{\left[M_{i}, M_{j}\right] } & =i c_{i j k} M_{k}, \\
{\left[R_{i}(t), R_{j}(t)\right] } & =i c_{i j k} R_{k}(t),
\end{aligned}
$$

are now real and totally antisymmetric in $i, j$, and $k$. We may still perform real rotations in the $N$-dimensional space of the $R_{i}$ or the $M_{i}$. Suppose, after performing such a rotation, that we can split the $R_{i}$ into two sets that commute with each other. Then our algebra is the direct sum of two commuting algebras. We continue this process until no further splitting is possible, even after performing rotations. The algebra has then been expressed as the direct sum of simple algebras. All the simple algebras have been listed by Cartan..$^{40}$ Besides the trivial one-dimensional algebra of $U(1)$ (which is not included by the mathematicians), there are the three-dimensional algebra of $S U(2)$, the eight-dimensional algebra of $S U(3)$, and so forth.

Now let us construct the currents of the operators $R_{i}$. We consider the gauge transformation of the second kind

$$
\psi_{A} \rightarrow \psi_{A}(\mathbf{x}, t)-i \Lambda_{i}(\mathbf{x}, t)\left[R_{i}(t), \psi_{A}(\mathbf{x}, t)\right],
$$

and ask what change it induces in the Lagrange density $L$. There will be a term in $\Lambda_{i}$ and a term in $\partial_{\alpha} \Lambda_{i}$, so adjusted $^{17}$ that the total change is just the divergence of a four-vector:

$L \rightarrow L(\mathbf{x}, t)-\partial_{\alpha} \Lambda_{i}(\mathbf{x}, t) R_{i \alpha}(\mathbf{x}, t)-\Lambda_{i}(\mathbf{x}, t) \partial_{\alpha} R_{i \alpha}(\mathbf{x}, t)$.

We define $R_{i \alpha}$ to be the current of $R_{i}$. It can be shown that $R_{i}$ is in fact given by the relation

$$
R_{i}=-i \int R_{i 4} d^{3} x
$$

Now if, for constant $\Lambda_{i}$, the whole Lagrangian is invariant under $R_{i}$, then the term in $\Lambda_{i}$ in (A4) must vanish; we have $\partial_{\alpha} R_{i \alpha}=0$. In other words, if there is exact symmetry under $R_{i}$, the current $R_{i \alpha}$ is conserved.

If there is a noninvariant part of $L$ with respect to the symmetry operation $R_{i}$, then the current will not be conserved. By hypothesis, the noninvariant term (call it $u$ ) contains no gradients. Therefore, the effect

${ }^{40} \mathrm{E}$. Cartan, Sur la Structure des groupes de transformations finis et continus, thèse (Paris, 1894; 2nd ed., 1933). 
of the transformation (A3) for constant $\Lambda_{i}$ will be simply to add a term $-i \Lambda_{i}\left[R_{i}, u\right]$ to the Lagrangian density. We have, then, using (A4), the result

$$
\partial_{\alpha} R_{i \alpha}(\mathbf{x}, t)=i\left[R_{i}(t), u(\mathbf{x}, t)\right] .
$$

Since $u$ contains no gradients, it is not only the noninvariant term in the Lagrangian density, but also the negative of the noninvariant term in the Hamiltonian density. The invariant part of $H$ evidently commutes with $R_{i}$. Thus we have

$$
\partial_{\alpha} R_{i \alpha}(\mathbf{x}, t)=-i\left[R_{i}(t), H(\mathbf{x}, t)\right] .
$$

By considering the transformation properties of $H$ under commutation with the algebra, we generate the divergences of all the currents. The formula obtained by integrating (A6) over space is, of course, very familiar:

$$
\dot{R}_{i}=\int \partial_{\alpha} R_{i \alpha} d^{3} x=-i\left[R_{i}, \int H d^{3} x\right]
$$

